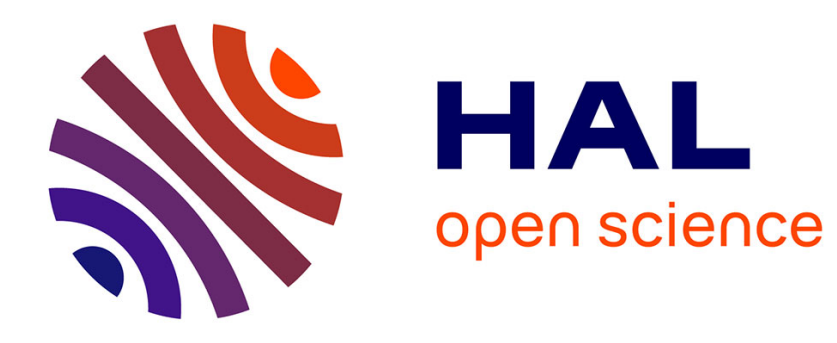

\title{
Growth and shape control of disks by bending and extension
}

\author{
Keith A. Seffen, Corrado Maurini
}

\section{To cite this version:}

Keith A. Seffen, Corrado Maurini. Growth and shape control of disks by bending and extension. Journal of the Mechanics and Physics of Solids, 2013, 61 (1), pp.190 - 204. 10.1016/j.jmps.2012.08.003 . hal-01502995

\section{HAL Id: hal-01502995 \\ https://hal.sorbonne-universite.fr/hal-01502995}

Submitted on 31 Jul 2017

HAL is a multi-disciplinary open access archive for the deposit and dissemination of scientific research documents, whether they are published or not. The documents may come from teaching and research institutions in France or abroad, or from public or private research centers.
L'archive ouverte pluridisciplinaire HAL, est destinée au dépôt et à la diffusion de documents scientifiques de niveau recherche, publiés ou non, émanant des établissements d'enseignement et de recherche français ou étrangers, des laboratoires publics ou privés. 


\title{
Growth and Shape Control of Disks by Bending and Extension
} Keith A Seffen

Department of Engineering, University of Cambridge, Trumpington Street, Cambridge, CB2 1PZ, United Kingdom Corrado Maurini

Institut Jean Le Rond d'Alembert, Université Pierre et Marie Curie, Paris 6, France

\begin{abstract}
Differential growth of thin elastic bodies furnishes a surprisingly simple explanation of the complex and intriguing shapes of many biological systems, such as plant leaves and organs. Similarly, inelastic strains induced by thermal effects or active materials in layered plates are extensively used to control the curvature of thin engineering structures. Such behaviour inspires us to distinguish and to compare two possible modes of differential growth not normally compared to each other, in order to reveal the full range of out-of-plane shapes of an initially flat disk. The first growth mode, frequently employed by engineers, is characterized by direct bending strains through the thickness, and the second mode, mainly apparent in biological systems, is driven by extensional strains of the middle surface. When each mode is considered separately, it is shown that buckling is common to both modes, leading to bistable shapes: growth from bending strains results in a double-curvature limit at buckling, followed by almost developable deformation in which the Gaussian curvature at buckling is conserved; during extensional growth, out-of-plane distortions occur only when the buckling condition is reached, and the Gaussian curvature continues to increase. When both growth modes are present, it is shown that, generally, larger displacements are obtained under in-plane growth when the disk is relatively thick and growth strains are small, and vice versa. It is also shown that shapes can be mono-, bi-, tri- or neutrally stable, depending on the growth strain levels and the material properties: furthermore, it is shown that certain combinations of growth modes result in a free, or natural, response in which the doubly-curved shape of disk exactly matches the imposed strains. Such diverse behaviour, in general, may help to realise more effective actuation schemes for engineering structures.
\end{abstract}




\section{Nomenclature}

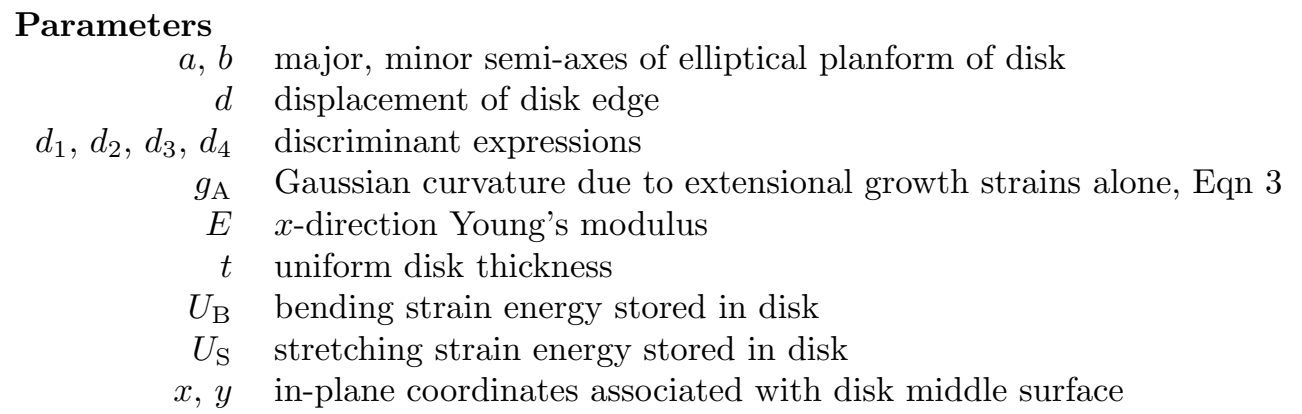

\section{Major symbols}

$\alpha \quad$ relative shear modulus, equal to $(1-\nu) / 2$ for isotropic behaviour

$\beta y$-direction specific Young's modulus, equal to unity for isotropic behaviour

$\gamma \quad$ bending growth effectiveness (maximum value of 2 )

$\epsilon$ middle surface strain tensor

$\epsilon_{\mathrm{A}} \quad$ middle surface growth strain tensor

$\epsilon_{\mathrm{E}} \quad$ elastic components of strain, equal to $\epsilon-\epsilon_{\mathrm{A}}$

$\bar{\epsilon}$ maximum growth strain

$\kappa$ curvature tensor

$\kappa_{\mathrm{A}} \quad$ curvature tensor due to bending growth strains

$\kappa_{\mathrm{E}}$ elastic components of curvature, equal to $\kappa-\kappa_{\mathrm{A}}$

$\nu$ Poisson's ratio

$\mu$ linearising parameter for governing equations of deformation, Eqn 7(d)

$\phi \quad$ material and geometry parameter for disk

$\eta$ relative weighting between bending growth $(= \pm 1)$ and extensional growth $(=0)$

\section{Other}

det $\kappa \quad$ Gaussian curvature due to bending, equal to $\kappa_{x} \kappa_{y}-\kappa_{x y}^{2}$

$\bar{\kappa}$ dimensionless curvature, equal to $\kappa a^{2} / t$

$\bar{g}_{\mathrm{A}}$ dimensionless Gaussian curvature due to extensional growth strains, equal to $g_{\mathrm{A}} a^{4} / t^{2}$

$\mathrm{A}, \mathrm{E}$ subscripts for growth and elastic properties, respectively 


\section{Introduction}

Morphogenesis considers the evolution and governance of shape in biological structures, and recent studies have identified the importance of mechanical effects and constraints on the origins of complex shapes under relatively simple driving forces (Dumais and Steele 2000). Many such structures have thin membranes or shells for reasons of geometrical efficiency and biological expediency, and the mechanics of these components provides essential insight into how growth proceeds. For example, instabilities such as local buckling of closed shells and membrane wrinkling simulate unique features of developing cell and organ growth in vitro as well as skin deterioration (Goriely and Ben Amar 2005, Cerda and Mahadevan 2003) - an excellent overall review is given by Chen and Yin (2010); the interplay between evolving shape and residual stresses in open strips describes the bent and twisted profiles of long leaves, kelp and seed pods (Liang and Mahadevan 2009, Koehl et al. 2008, Forterre and Dumais 2011, Armon et al. 2011); dynamical "snap-through" of a shell cap can explain the rapid closure of the Venus Flytrap without muscular intervention (Forterre et al. 2005); and the elongation of cell tips in pollen (Campas and Mahedvan 2009, Aouar et al. 2010) behaves like a so-called propagating instability (Kyriakides 1994), which - we surmise - expedites the growth of highly deformed areas at much lower driving forces than expected.

This paper focuses on recent studies concerning the complex shapes attained by an initially flat, unconstrained surface when prescribing inelastic deformations that model growth phenomena in biological systems (Klein et al. 2007, Dervaux and Ben-Amar 2008, Dervaux et al. 2009). Despite its apparent simplicity, the problem encapsulates a fundamental responsive mode in deforming shells, in which the actual deformations differ from the prescribed deformations because of the interplay between bending and extensional effects. Often, and correctly, biologists and physicists assume that, because the surface is relatively thin, growth produces extensional strains, which are uniform through the thickness. The strains induced by growth may be unreachable in practice because of geometrical incompatibility and, as carefully described by Klein et al. (2007), their non-uniform distribution within the middle surface of shell correspond to non-Euclidean metrics of the surface, i.e. to metrics unattainable by surfaces embedded in a three-dimensional Euclidean space. In response, the surface develops a state of self-stress whilst deforming elastically to accommodate the incompatible growth strains, and this can lead to interesting buckling phenomena. Although the shape is controlled mainly by the mechanical properties, there is some evidence that the degree of difference has a genetic component in practice (Nath et al. 2003).

At the same time, controlling the shape of a surface and conceiving "morphing" structures is an active research subject in mechanical engineering, where active materials and multi-physical couplings (e.g. piezoelectric, thermoelastic, hygroscopic, plasticity) provide the means to induce controllable inelastic strains analogous to growth. On the basis of linear theories, engineers have long assumed that large displacements in thin shells are wrought most effectively by bending actuation composed of differential through-thickness strains: a familiar example is a bimetallic plate that curves directly out-of-plane under a rise in ambient temperature (Wittrick et al. 1953). However, large displacements in shells entail a coupling between bending and in-plane extension, which ultimately limits the authority of bending actuation. For thin surfaces, there is potentially a choice for engineers in the mode of actuation - the so-called "actuation paradigm" mentioned by Modes et al. (2011) - if extensional actuation can provide an efficient alternative to bending. Thus, research on shape control of structures may benefit from this study, for out-of-plane deformation of shells using in-plane actuation exclusively is only recently garnering attention and may prove competitive compared to traditional bilayer (bending) methods. There are also implications for processes involving plastically working thin-walled engineering materials where, like biological growth, the deformation is irreversible and usually evolves incrementally: residual stresses confer the desired shape of shell - which differs from the final shape because the imposed deformation cannot proceed without constraint.

In this paper, growth and actuation are treated together as simple inelastic strain distributions simultaneously conferred upon a flat, stress-free disk. Our primary aim is to establish the differences in the out-of-plane shapes arising from extensional and bending modes - whether by growth or from actuationwhen they act separately and when both are present. The latter "interaction" of modes is entirely novel 
because it has not been reported formally in the literature, and is essential for the reasons described above. We consider this interaction in two ways, by prescribing modes that are independent and then coupled by a common maximum growth strain. The first enables us to ascertain the general behaviour, e.g. the stability complexion, and the second allows pertinent details to be found, such as the maximum shape change etc.

The study of the interaction of growth modes is underpinned by their individual responses, and these have been eschewed, in part, by others. For example, the first author reports on the bending actuation of a disk (Seffen and Guest 2011), and the principle underlying extensional growth of a flat disk is confirmed in numerical simulations by Klein et al. (2007). Inevitably, we repeat-and declare - this work as part the broader presentation of this paper, but the imperative and, hence, novelty of this study lies with discussing the relative shape-control efficiency of the two growth modes by describing all possible shapes of the disk in closed form. Most studies simulating morphogenesis extract meaningful trends from exhaustive computations because the problem details are not amenable to a compact theoretical analysis without a loss of accuracy. A flat disk is geometrically very simple but analysis of its growth response is non-trivial, resulting in a virtual dearth of compact formulae describing key relationships. Adopting the same approach in Seffen and Guest (2011) and Fernandes et al. (2010), we manage a theoretical approach by assuming that first-order (uniform) changes in shape occur, and these reasonably follow if the growth strains themselves correlate to uniform imposed curvatures, even if the current shape of disk does not match this "target" shape. To this end, we extend earlier work from the authors (Seffen and Guest 2011, Fernandes et al. 2010) to explicitly include and discuss the effect of extensional growth, and we complement matters by performing a non-linear, large displacement finite element analysis, which makes no assumptions about the uniformity of shape and the order of magnitude of the geometrical non-linearities. The comparison of numerical and analytical results clearly proves the (surprisingly) moderate sacrifice in the accuracy of shape and the pertinence of our strongly simplified model for the analysis of uniform bending and/or extensional growth. If instead the growth strains are non-uniform, the shape of disk would be described by higher-order modes throughout, as observed by Klein et al. (2007) in their experiments where practical arrangements do not confer a uniform change; their results would naturally extend the results here but are the subject of further work.

Section 2 describes the geometrical definitions and assumptions in detail, in order to establish the meaning of kinematical concepts not used by all researchers. In particular, we carefully discuss how the target shape of disk and its actual shape differ in view of strict compatibility rules. This is not often clear to engineers, and importantly, it allows us to incorporate the effect of extensional growth strains into earlier formulations on bending growth alone, developed separately by both authors, with relative ease (Seffen 2007, Vidoli and Maurini 2008, Fernandes et al. 2010). This formulation is sketched in Section 3 where the final governing equations of shape are presented and compared to expressions in previous studies for the sake of clarity. Section 4 then considers "single" mode solutions, where extension and bending growth are treated separately, and the predictions of shape are compared to a non-linear finite element analysis using simple distributions of growth strains. The modes are then compared together by setting a common limit on the maximal growth strain, as if they had the same constitutive growth capabilities. Section 5 formally presents "double" mode growth in which extensional and bending growth strains are both present. Their interaction is first plotted as a regime map, which identifies regions with a particular degree of stability. From a design perspective, this map can be interrogated quickly without calculating the exact shape of disk. The largest possible displacements arising from the relative influence of each mode is then revealed as another map and, finally, we consider how the associated strain energy varies. In Section 6, we refer briefly to the case of natural growth, where the shape induced by both modes is followed exactly by the disk so that as the disk deforms, no strain energy is stored but it retains its stiffness, and this opens up interesting possibilities for developing large-displacement actuators that are structurally robust. Section 7 concludes this study. 


\section{Definitions of Geometry and Assumptions}

The disk is initially flat, it is thin with a constant (uniform) thickness and has an elliptical planform of major- and minor semi-axes, $a$ and $b$. During growth or actuation, in-plane strains develop, leading to a change of the outwards shape, mainly in the displacements normal to the disk surface when the disk is very thin. These displacements are assumed to be shallow but finite so that equilibrium is related by the deformed configuration of disk. The underlying variation of strains through the disk thickness can be condensed into properties of the middle surface alone, namely tensors of in-plane strain, $\epsilon$, and of out-of-plane curvature, $\kappa$. These tensors describe the current shape of the disk and, correspondingly, Gauss's Theorema Egregium suggests a connection between the variations of the strain tensor and the Gaussian curvature, denoted by $G(\epsilon)$. Writing the strains in terms of direct components, $\epsilon_{x}$ and $\epsilon_{y}$, and a shear strain, $\gamma_{x y}$, for orthogonal coordinates $x$ and $y$ in the original plane of disk, the linearised form reads as (Calladine 1983)

$$
G(\epsilon)=-\frac{\partial^{2} \epsilon_{x x}}{\partial y^{2}}-\frac{\partial^{2} \epsilon_{y y}}{\partial x^{2}}+\frac{\partial^{2} \gamma_{x y}}{\partial x \partial y}
$$

and, recognising that the Gaussian curvature is set by det $\kappa=\kappa_{x} \kappa_{y}-\kappa_{x y}^{2}$ for ordinary curvatures, $\kappa_{x}$ and $\kappa_{y}$, and twisting curvature, $\kappa_{x y}$, the connection is succinctly written as

$$
G(\epsilon)=\operatorname{det} \kappa
$$

The current shape depends on the response of the disk to so-called inelastic deformations conferred by growth or actuation processes, denoted by subscript "A". These processes are assumed to produce planar strain distributions through the thickness, which may be decoupled into components of middle surface strains, $\epsilon_{\mathrm{A}}$, and curvatures, $\kappa_{\mathrm{A}}$. Here, the subscript "A" collectively denotes growth or actuation but importantly, the distinction between extensional and bending effects has been set-separately from the physical origin of each process that causes them. We do note that either of $\epsilon_{\mathrm{A}}$ or $\kappa_{\mathrm{A}}$ may be zero, or both may occur simultaneously and may be coupled to each other. For the sake of generality, we shall assume that they are separately prescribed, which has implications for the elastic response of the disk. By definition, $\epsilon_{\mathrm{A}}$ and $\kappa_{\mathrm{A}}$ are connected to Gaussian curvature using the same linearised approximations as for the current shape before, i.e. they produce components $G\left(\epsilon_{\mathrm{A}}\right)$ and $\operatorname{det}\left(\kappa_{\mathrm{A}}\right)$; but we cannot say that $G\left(\epsilon_{\mathrm{A}}\right)$ is equal to $\operatorname{det}\left(\kappa_{\mathrm{A}}\right)$ since $\epsilon_{\mathrm{A}}$ and $\kappa_{\mathrm{A}}$ are independent. Alternatively, if the current shape follows from setting $\epsilon=\epsilon_{\mathrm{A}}$ and $\kappa=\kappa_{\mathrm{A}}$, Eqn 2 is not satisfied and the target shape defined by $\epsilon_{\mathrm{A}}$ and $\kappa_{\mathrm{A}}$ cannot be reached. Elastic components of deformation must be induced and, again, there are two middle surface quantities denoted by subscript "E", $\epsilon_{\mathrm{E}}$ and $\kappa_{\mathrm{E}}$, which define the difference between the current and target shapes according to $\epsilon_{\mathrm{E}}=\epsilon-\epsilon_{\mathrm{A}}$ and $\kappa_{\mathrm{E}}=\kappa-\kappa_{\mathrm{A}}$. The degree of difference in both will depend on the final equilibrium conditions, which are most simply addressed by considering the strain energy stored in the disk.

In order to enable an approach soluble in closed form, we further simplify the nature of the inelastic terms. Foremost, we assume that $\operatorname{det} \kappa_{\mathrm{A}}$ and $G\left(\epsilon_{\mathrm{A}}\right)$ do not vary with $x$ or $y$ within the disk, thereby resulting in uniform Gaussian curvatures. Recent studies (Freund 2000, Guest and Pellegrino 2006, Seffen and McMahon 2007, Seffen 2007, Vidoli and Maurini 2008) have indicated that the disk responds in a similar manner by adopting uniform curvatures, which reduces the calculations substantially. This is not, however, a restrictive assumption in view of common growth or actuation processes. For example, a heated bimetallic disk attempts to curve equally and uniformly in all directions, and a rolled sheet can be cylindrically curved into a circular arc, so we specify separate components of bending growth, $\kappa_{x \mathrm{~A}}$ and $\kappa_{y \mathrm{~A}}$, in the $x$ and $y$ directions, respectively, and a uniform twisting growth, $\kappa_{x y \mathrm{~A}}$, such that $\operatorname{det} \kappa_{\mathrm{A}}=\kappa_{x \mathrm{~A}} \kappa_{y \mathrm{~A}}-\kappa_{x y \mathrm{~A}}^{2}$, a constant. In the case of extensional growth, uniformity stems from a quadratic strain field since $G\left(\epsilon_{\mathrm{A}}\right)$ has second-order derivatives of the components of $\epsilon_{\mathrm{A}}$ : indeed, if we assume that $\epsilon_{\mathrm{A}}$ is isotropic and proportional to $x^{2}+y^{2}$, then a parallel form of Eqn 2 yields

$$
G\left(\epsilon_{\mathrm{A}}\right)=-\frac{\partial^{2} \epsilon_{\mathrm{A}}}{\partial y^{2}}-\frac{\partial^{2} \epsilon_{\mathrm{A}}}{\partial x^{2}}=g_{\mathrm{A}}, \text { a constant }
$$


where $g_{\mathrm{A}}$ replaces $G\left(\epsilon_{\mathrm{A}}\right)$ for simplicity. Quadratic strain fields can arise often in Nature because of the way in which nutrients for growth are absorbed through the surface. For example, long strips of kelp are more exposed on their outer edges in fast-flowing rivers, resulting in a cross-wise variation of growth strain that is approximately parabolic (Koehl et al. 2008); finding ways to actuate a quadratic strain field in an engineering structure remains to be realised, and efforts may be garnered by the results of this study, in which we chose idealised strain fields for the simplest, quantitative comparison between both modes.

\section{Strain Energy Formulation and Governing Equations}

The disk is thin and homogenous, and the material response is linear because strains remain small despite relatively large displacements. The constitutive elastic behaviour is taken to be orthotropic following Seffen (2007), who considered shells without growth strains but which can be adapted to include the definitions of $\epsilon_{\mathrm{A}}$ and $\kappa_{\mathrm{A}}$ from Section 2 by replacing the elastic deformations with $\epsilon_{\mathrm{E}}=\epsilon-\epsilon_{\mathrm{A}}$ and $\kappa_{\mathrm{E}}=\kappa-\kappa_{\mathrm{A}}$. Correspondingly, the strain energy stored in the disk can be found by considering separate components of strain energy density in bending and in extension, which are integrated over the entire middle surface. A conservative behaviour is also assumed so that the disk returns to being flat when the growth strains are "switched off", and no external loads are applied to the disk, generally.

The bending strain energy bending density has a quadratic form equal to $(1 / 2) \kappa_{\mathrm{E}}^{\mathrm{T}} D^{\mathrm{T}} \kappa_{\mathrm{E}}$, where $D$ is a $3 \times 3$ matrix comprising elements of bending and torsional rigidities - see Vidoli and Maurini (2008) for example - and $\kappa_{\mathrm{E}}$ is the vector of elastic curvatures, $\left[\begin{array}{llll}\kappa_{x \mathrm{E}} & \kappa_{y \mathrm{E}} & 2 \kappa_{x y \mathrm{E}}\end{array}\right]^{\mathrm{T}}$, using Voight notation. Because the elastic curvatures are uniform throughout the disk, the total bending component, denoted by $U_{\mathrm{B}}$, premultiplies the density by the elliptical planform area of the disk, and the final form reads in terms of the current curvatures, $\kappa$, and target curvatures, $\kappa_{\mathrm{A}}$, as:

$$
U_{\mathrm{B}}=\frac{\pi a b E t^{3}}{24\left(1-\nu^{2} / \beta\right)}\left[\left(\kappa_{x}-\kappa_{x \mathrm{~A}}\right)^{2}+2 \nu\left(\kappa_{x}-\kappa_{x \mathrm{~A}}\right)\left(\kappa_{y}-\kappa_{y \mathrm{~A}}\right)+\beta\left(\kappa_{y}-\kappa_{y \mathrm{~A}}\right)^{2}+4 \alpha\left(\kappa_{x y}-\kappa_{x y \mathrm{~A}}\right)^{2}\right]
$$

$E$ is the Young's modulus in the $x$-direction and $\beta$ is the relative modulus in the $y$-direction; $\alpha$ is the relative shear modulus and $\nu$ relates to the Poisson ratio. For the isotropic case, $\beta=1$ and $\alpha=(1-\nu) / 2$, otherwise, specifying general values allows us to model a range of orthotropic materials. Mathematically, $-1<\nu<1$ and $\beta>\nu^{2}$ for positive definiteness of the constitutive material matrix and, from a practical viewpoint, materials are generally not weaker in shear so $\alpha$ is only ever equal to, or larger than, its isotropic value.

The extensional strain energy density also has a quadratic form, which is first written in terms of $\epsilon_{\mathrm{E}}$, but which can be expressed commensurately in terms of curvature by the following route. Recall that the assumption of uniform curvatures implies a quadratic variation in the expected distribution of current strains, $\epsilon$, via Eqns 1 and 2. To find the exact variation, the components of $\epsilon$ in Eqn 1 are replaced by $\epsilon_{\mathrm{E}}+\epsilon_{\mathrm{A}}$, and the differential operator turns $\epsilon_{\mathrm{A}}$ into $g_{\mathrm{A}}$ on the right-hand side. The elastic strains, $\epsilon_{\mathrm{E}}$, are then replaced by elastic stresses, by inverting the constitutive elasticity matrix to find the required relationships. The stresses are written in terms of an Airy stress function, $\Phi$, as performed in Seffen (2007) so that Eqn 1 now becomes a fourth-order differential equation with a constant "forcing" term equal to $\operatorname{det} \kappa-g_{\mathrm{A}}$. A polynomial for $\Phi$ can be selected so that differential equation is satisfied whilst upholding the boundary conditions of zero normal force and shear force on the periphery of the disk. The stresses and hence, strains are determined and substituted back into the strain energy density, and this expression is integrated over the disk area to reveal a total component, $U_{\mathrm{S}}$, equal to

$$
U_{\mathrm{S}}=\frac{E t \pi a^{5} b^{5}}{48\left(1-\nu^{2} / \beta\right)} \frac{\alpha\left(\beta-\nu^{2}\right)}{3 a^{4} \beta \alpha+3 b^{4} \alpha+a^{2} b^{2}\left[\beta-\nu^{2}-2 \nu \alpha\right]}\left(\operatorname{det} k-g_{\mathrm{A}}\right)^{2}
$$

The total strain energy, $U=U_{\mathrm{S}}+U_{\mathrm{B}}$, is made dimensionless by defining 


$$
\bar{U}=U \cdot \frac{12 a^{3}\left(1-\nu^{2} / \beta\right)}{E \pi b t^{5}}, \quad \bar{\kappa}=\kappa \cdot \frac{a^{2}}{t}, \quad \rho=\frac{a}{b}, \quad \phi=\frac{\alpha\left(\beta-\nu^{2}\right)}{6 \rho^{4} \beta \alpha+6 \alpha+2 \rho^{2}\left[\beta-\nu^{2}-2 \nu \alpha\right]}
$$

so that

$$
\bar{U}=\frac{\phi}{2}\left(\operatorname{det} \bar{k}-\bar{g}_{\mathrm{A}}\right)^{2}+\frac{1}{2}\left[\left(\bar{\kappa}_{x}-\bar{\kappa}_{x \mathrm{~A}}\right)^{2}+2 \nu\left(\bar{\kappa}_{x}-\bar{\kappa}_{x \mathrm{~A}}\right)\left(\bar{\kappa}_{y}-\bar{\kappa}_{y \mathrm{~A}}\right)+\beta\left(\bar{\kappa}_{y}-\bar{\kappa}_{y \mathrm{~A}}\right)^{2}+4 \alpha\left(\bar{\kappa}_{x y}-\bar{\kappa}_{x y \mathrm{~A}}\right)^{2}\right]
$$

where $\operatorname{det} \bar{k}=\bar{\kappa}_{x} \bar{\kappa}_{y}-\bar{\kappa}_{x y}^{2}$ : note that $\phi=\left(1-\nu^{2}\right) / 16$ for a circular isotropic disk. Since $\bar{U}$ is algebraic, statical equilibrium configurations are obtained by setting $\partial \bar{U} / \partial \bar{\kappa}=0$ for each of $\bar{\kappa}_{x}, \bar{\kappa}_{y}$ and $\bar{\kappa}_{x y}$. The resulting general governing equations are non-linear coupled expressions of the current shape, which can be written in a slightly decoupled form as:

$$
\begin{array}{cc}
\bar{\kappa}_{x}+\mu \bar{\kappa}_{y}=\bar{\kappa}_{x \mathrm{~A}}+\nu \bar{\kappa}_{y \mathrm{~A}} \quad(\mathrm{a}) ; \quad \mu \bar{\kappa}_{x}+\beta \bar{\kappa}_{y}=\nu \bar{\kappa}_{x \mathrm{~A}}+\beta \bar{\kappa}_{y \mathrm{~A}} \quad \text { (b); } \\
(2 \alpha-\mu+\nu) \bar{\kappa}_{x y}=2 \alpha \bar{\kappa}_{x y \mathrm{~A}} \quad \text { (c); } \quad \mu=\nu+\phi\left(\bar{\kappa}_{x} \bar{\kappa}_{y}-\bar{\kappa}_{x y}^{2}-\bar{g}_{\mathrm{A}}\right)
\end{array}
$$

These are similar to the expressions furnished by Seffen and Guest (2011) for a pre-stressed disk in which growth is analogous to plastic bending, but only because of how they are written above. Here, we now account for extensional growth via $g_{\mathrm{A}}$ in $\mu$ everywhere, which results in new solutions both for extensional growth alone and when both modes are present.

Despite their compactness, any solution to these expressions is generally formidable but which may be guided in the following way. Equations $7(\mathrm{a})$-(c) are, first, explicitly soluble for $\bar{\kappa}_{x}, \bar{\kappa}_{y}$ and $\bar{\kappa}_{x y}$ in terms of the Gaussian curvature parameter, $\mu$, and the components of $\bar{\kappa}_{\mathrm{A}}$. Once found, these expressions are substituted back into $\mu$ in Eqn $7(\mathrm{~d})$, which also includes $\bar{g}_{\mathrm{A}}$, and the following seven-order polynomial emerges after tidying up:

$$
\begin{gathered}
\phi\left\{\left[\left(\beta+\mu^{2}\right)\left(\bar{\kappa}_{x \mathrm{~A}}+\nu \bar{\kappa}_{y \mathrm{~A}}\right)\left(\nu \bar{\kappa}_{x \mathrm{~A}}+\beta \bar{\kappa}_{y \mathrm{~A}}\right)-\mu\left(\nu \bar{\kappa}_{x \mathrm{~A}}+\beta \bar{\kappa}_{y \mathrm{~A}}\right)^{2}-\mu \beta\left(\bar{\kappa}_{x \mathrm{~A}}+\nu \bar{\kappa}_{y \mathrm{~A}}\right)^{2}\right](2 \alpha-\mu+\nu)^{2}\right. \\
\left.-4 \alpha^{2} \bar{\kappa}_{x y \mathrm{~A}}^{2}\left(\beta-\mu^{2}\right)^{2}\right\}-\left(\phi \bar{g}_{\mathrm{A}}+\mu-\nu\right)(2 \alpha-\mu+\nu)^{2}\left(\beta-\mu^{2}\right)^{2}=0
\end{gathered}
$$

The roots in $\mu$ of this characteristic equation determine the curvatures for any value and combination of the growth strains, as well as specified material and geometrical properties, and are typically found by numerical solution. Importantly, the roots must be real for the resulting shape to be physically possible.

Some roots are admissible in closed form, as performed in Seffen (2007) for initially curved, stress-free disks, but only after simplifying the characteristic equation for the sake of tractability. This entails a coupling of some of the independent terms - the growth strains here, so not all possible solutions can be examined. However, the descriptions of shape are vital in expounding the evolution of growth, and therefore we choose to seek out as many closed-form solutions. When considering Eqn 8, note that it can be simplified immediately by setting the repeating terms, $\left(\beta-\mu^{2}\right)$ and $(2 \alpha-\mu+\nu)$, equal to zero, which then sets conditions upon the remaining terms for overall satisfaction. These same conditions fortuitously allow the original governing equations to be solved elegantly by inspection, to reveal another solution regime in which $\mu$ varies rather than being a constant root value. These cases are now treated in turn.

$$
\text { (a.i) } \mu=2 \alpha+\nu, \mu \neq \pm \sqrt{\beta}
$$

This solution is only viable when $\bar{\kappa}_{x y \mathrm{~A}}$ is equal to zero, otherwise the characteristic equation and, ultimately, Eqn 7(c) are not satisfied. Correspondingly, the twisting curvature is not specified directly. Solutions for $\bar{\kappa}_{x}$ and $\bar{\kappa}_{y}$ must be obtained first from solving Eqns $7(\mathrm{a})$ and (b) together using $\mu=2 \alpha+\nu$, before substituting back into Eqn $7(\mathrm{~d})$, in order to solve for $\bar{\kappa}_{x y}$. In this case, the combination of growth strain values 
must enable $\bar{\kappa}_{x y}$ to be real, and a separate study has shown that the shear modulus must be less than its isotropic value for stable shapes; however, as noted before, practical materials do not usually behave in this way, and we do not pay any further attention to these twisted solutions.

$$
\text { (a.ii) } \mu= \pm \sqrt{\beta}, \mu \neq 2 \alpha+\nu
$$

In this case, there are two root values of $\mu$, either $\sqrt{\beta}$ or $-\sqrt{\beta}$, and these predicate separate relationships upon the growth strains, which are represented together via the " \pm " sign. Consider first when $\bar{\kappa}_{x y \mathrm{~A}}$ is set equal to zero so that there is no twisting curvature from Eqn 7(c). Satisfaction of the characteristic equation is now obtained by setting the term inside square brackets equal to zero. After some manipulation, this produces the rather compact requirement:

$$
\pm \sqrt{\beta}\left(\nu \bar{\kappa}_{x \mathrm{~A}}+\beta \bar{\kappa}_{y \mathrm{~A}}\right)=\bar{\kappa}_{x \mathrm{~A}}+\nu \bar{\kappa}_{y \mathrm{~A}} \Rightarrow \bar{\kappa}_{y \mathrm{~A}}=\frac{\bar{\kappa}_{x \mathrm{~A}}}{ \pm \sqrt{\beta}}
$$

which is practicable and enables a wide range of solution shapes. Using either of Eqns $7(\mathrm{a})$ or (b) and the definition of $\mu$, it may be shown that:

$$
\bar{\kappa}_{x} \pm \sqrt{\beta} \bar{\kappa}_{y}=\left(1 \mp \frac{\nu}{\sqrt{\beta}}\right) \bar{\kappa}_{x \mathrm{~A}}, \quad \frac{ \pm \sqrt{\beta}-\nu}{\phi}=\bar{\kappa}_{x} \bar{\kappa}_{y}-\bar{g}_{\mathrm{A}}
$$

These are two soluble equations in $\bar{\kappa}_{x}$ and $\bar{\kappa}_{y}$, which can be re-stated in terms of either parameter: we choose $\bar{\kappa}_{x}$ and eliminate $\bar{\kappa}_{y}$, to reveal

$$
\mu= \pm \sqrt{\beta}, \bar{\kappa}_{y} \neq \bar{\kappa}_{x}: \bar{\kappa}_{y \mathrm{~A}}=\frac{\bar{\kappa}_{x \mathrm{~A}}}{ \pm \sqrt{\beta}} \Rightarrow \phi \bar{\kappa}_{x}^{2}-\phi\left[1 \pm \frac{\nu}{\sqrt{\beta}}\right] \bar{\kappa}_{x \mathrm{~A}} \bar{\kappa}_{x}+\sqrt{\beta}\left[\sqrt{\beta} \mp \nu \pm \phi \bar{g}_{\mathrm{A}}\right]=0
$$

The final quadratic equation is to be read as two equations, depending on whether $\bar{\kappa}_{y \mathrm{~A}}=\bar{\kappa}_{x \mathrm{~A}} / \sqrt{\beta}$ or $\bar{\kappa}_{y \mathrm{~A}}=-\bar{\kappa}_{x \mathrm{~A}} / \sqrt{\beta}$. The roots of either equation produce explicit expressions for $\bar{\kappa}_{x}$, which can be substituted back in to Eqn 10 to find the same for $\bar{\kappa}_{y}$, and this is presented momentarily.

For the case of $\bar{\kappa}_{x y \mathrm{~A}} \neq 0$, then $\bar{\kappa}_{x y}$ is uniquely defined by $2 \alpha \bar{\kappa}_{x y \mathrm{~A}} /(2 \alpha+\nu \mp \sqrt{\beta})$. Setting $\bar{\kappa}_{y \mathrm{~A}}=\bar{\kappa}_{x \mathrm{~A}} / \pm \sqrt{\beta}$ gives similar soluble expressions to Eqn 10 but where the product $\bar{\kappa}_{x} \bar{\kappa}_{y}$ is replaced by $\bar{\kappa}_{x} \bar{\kappa}_{y}-\bar{\kappa}_{x y}^{2}$. These can be solved in the same way but are considered no further: there is also little evidence of this type of growth strain in practice.

$$
\text { (a.iii) } \mu \neq \pm \sqrt{\beta}, \mu \neq 2 \alpha+\nu
$$

In this case, there are no obvious roots of the characteristic equation. However, if the twisting terms are zero and we retain $\bar{\kappa}_{y \mathrm{~A}}=\bar{\kappa}_{x \mathrm{~A}} / \pm \sqrt{\beta}$, then Eqns 7 may be solved in a very simple way. Specifically, $\bar{\kappa}_{x y}$ is zero via Eqn $7(\mathrm{c})$, and we note that Eqns $7(\mathrm{a})$ and (b) are multiples of each other when $\bar{\kappa}_{y}$ is set equal to $\bar{\kappa}_{x} / \pm \sqrt{\beta}$. Correspondingly, $\mu$ can be eliminated between these expressions, so that the curvature solutions do not depend on $\mu$, rather, they prescribe its value separately in Eqn $7(\mathrm{~d})$ quite unlike the previous cases of constant roots. Using either of Eqns 7(a) or (b) and (d), the intermediate step produces:

$$
\bar{\kappa}_{x}=\frac{\bar{\kappa}_{x \mathrm{~A}}(\sqrt{\beta} \pm \nu)}{\sqrt{\beta} \pm \mu}, \quad \mu=\nu+\phi\left(\frac{\bar{\kappa}_{x}^{2}}{ \pm \sqrt{\beta}}-\bar{g}_{\mathrm{A}}\right)
$$

and, after substituting for $\mu$ into the first expression, another solution regime is found, which reads as the following cubic equation in $\bar{\kappa}_{x}$ :

$$
\bar{\kappa}_{y}=\frac{\bar{\kappa}_{x}}{ \pm \sqrt{\beta}}: \bar{\kappa}_{y \mathrm{~A}}=\frac{\bar{\kappa}_{x \mathrm{~A}}}{ \pm \sqrt{\beta}} \Rightarrow \phi \bar{\kappa}_{x}^{3}+\sqrt{\beta}\left[\sqrt{\beta} \pm \nu \mp \phi \bar{g}_{\mathrm{A}}\right] \bar{\kappa}_{x}-\sqrt{\beta}[\sqrt{\beta} \pm \nu] \bar{\kappa}_{x \mathrm{~A}}=0
$$


Equations 11 and 13 are specific governing equations because they describe one of the curvatures explicitly. They also depend on the same growth strain condition, namely $\bar{\kappa}_{y \mathrm{~A}}=\bar{\kappa}_{x \mathrm{~A}} / \sqrt{\beta}$, and so they apply equally to the disk. Obviously, the choice of internal sign declares the form of bending growth, but the prevailing solution regime offers the most stable shapes, and these are treated in detail in Sections 4 and 5.

$$
\text { (a.iv) } \mu= \pm \sqrt{\beta}, \mu=2 \alpha+\nu
$$

This case is similar to (a.i) merged with (a.ii), with the proviso on material properties that $\sqrt{\beta}=2 \alpha+\nu$ for positive values of constants: $\mu=-\sqrt{\beta}$ cannot apply. Thus, $\bar{\kappa}_{x}$ and $\bar{\kappa}_{y}$ are governed by Eqn 10 , and $\bar{\kappa}_{x y}$ follows from Eqn 7(d) and, once again, stable shapes occur only when the shear modulus is less than its isotropic value. Although we have noted that these twisted solutions are not of interest in general, the single case involving isotropic parameters presents a special case in which $\bar{\kappa}_{x y}=0$, and this emerges later in the detailed solutions.

\section{(b) Shape stability}

The stability of shape is assessed by substituting the expressions for $\bar{\kappa}_{x}$ and $\bar{\kappa}_{y}$ (and $\bar{\kappa}_{x y}=0$ ) into the following generalised stiffness matrix of the disk, whose elements comprise $\partial^{2} \bar{U} / \partial \bar{\kappa}_{x}^{2}, \partial^{2} \bar{U} / \partial \bar{\kappa}_{x} \bar{\kappa}_{y}$ etc beforehand:

$$
\left[\begin{array}{ccc}
1+\phi \bar{\kappa}_{y}^{2} & \mu+\phi \bar{\kappa}_{y} \bar{\kappa}_{x} & -2 \phi \bar{\kappa}_{y} \bar{\kappa}_{x y} \\
\mu+\phi \bar{\kappa}_{x} \bar{\kappa}_{y} & \beta+\phi \bar{\kappa}_{x}^{2} & -2 \phi \bar{\kappa}_{x} \bar{\kappa}_{x y} \\
-2 \phi \bar{\kappa}_{x y} \bar{\kappa}_{y} & -2 \phi \bar{\kappa}_{x y} \bar{\kappa}_{x} & 4 \alpha-2(\mu-\nu)+4 \phi \bar{\kappa}_{x y}^{2}
\end{array}\right]
$$

Shapes are stable only if this matrix is positive definite - when its three eigenvalues are positive; if any is zero, the disk is neutrally stable in that mode; a negative eigenvalue produces an unstable equilibria. Note that $\bar{g}_{\mathrm{A}}$ features directly in this matrix via $\mu$, and the contribution of $\bar{\kappa}_{x \mathrm{~A}}$ or $\bar{\kappa}_{y \mathrm{~A}}$ is implied through the solution curvatures.

\section{(c) Summary of analysis}

We can determine the out-of-plane shape of a uniform elliptical disk subjected to growth strains, which foist a change in its Gaussian curvature by extensional and/or bending growth (or actuation processes), by focussing on the roots of a characteristic equation. These can be found numerically for any combination of growth strains but we choose, instead, to prescribe specific relationships between the bending components according to $\bar{\kappa}_{y \mathrm{~A}}=\bar{\kappa}_{x \mathrm{~A}} / \pm \sqrt{\beta}$ and $\bar{\kappa}_{y}=\bar{\kappa}_{x} / \pm \sqrt{\beta}$, but not at the same time, for solutions in closed-form. Because they are formulaic in nature, the character and stability becomes apparent despite the reduced range of possible growth strains, but this is more instructive and is the focus of the rest of this paper. We make one more restriction by setting $\beta=1$ for marginal convenience henceforth: this stipulates direct isotropy but it does not detract from the generality of results, for example, see Fernandes et al. (2010). We do retain an independent shear modulus, for its value does affect the stability of shapes as is now discussed, first, for single mode growth and then for interacting, or double mode, growth.

\section{Single Mode Growth}

(a) Extensional growth, $\bar{g}_{\mathrm{A}} \neq 0,\left(\bar{\kappa}_{x \mathrm{~A}}, \bar{\kappa}_{y \mathrm{~A}}\right)=0$

When the bending components, $\bar{\kappa}_{x \mathrm{~A}}$ and $\bar{\kappa}_{y \mathrm{~A}}$, are set equal to zero in Eqns 11 and 13 , three sets of closedform solutions are found. The first has $\left(\bar{\kappa}_{x}, \bar{\kappa}_{y}\right)=0$, and this is valid in either direction as $\bar{g}_{\mathrm{A}}$ increases or decreases, until $\bar{g}_{\mathrm{A}}$ reaches a critical value denoted as $\bar{g}_{\mathrm{A}}^{*}$ such that 


$$
\bar{g}_{\mathrm{A}}^{*}=\frac{\mp 1+\nu}{\phi}
$$

Since the magnitude of the Poisson ratio is never larger than unity, $\bar{g}_{\mathrm{A}}^{*}=(1+\nu) / \phi$ is positive and $\bar{g}_{\mathrm{A}}^{*}=$ $-(1-\nu) / \phi$ is negative. When $\left|\bar{g}_{\mathrm{A}}\right|<\left|\bar{g}_{\mathrm{A}}^{*}\right|$, the stability matrix can be verified as being positive definite for $\left(\bar{\kappa}_{x}, \bar{\kappa}_{y}\right)=0$. by

When $\bar{g}_{\mathrm{A}}$ reaches positive $\bar{g}_{\mathrm{A}}^{*}$, the disk now buckles into an out-of-plane mode of equal curvatures defined

$$
\bar{\kappa}_{x}=\bar{\kappa}_{y}= \pm \sqrt{\bar{g}_{\mathrm{A}}-\frac{(1+\nu)}{\phi}}
$$

The \pm sign in this second set indicates that the disk can deflect upwards or downwards - it is able to occupy both in a bistable manner, which is confirmed after substituting these expressions into Eqn 14 provided $\bar{g}_{\mathrm{A}} \geq(1+\nu) / \phi$.

The third set arises when $\bar{g}_{\mathrm{A}}$ is less than the critical negative value, $\bar{g}_{\mathrm{A}}^{*}=-(1-\nu) / \phi$. The disk has buckled again but its shape has equal and opposite curvatures:

$$
\bar{\kappa}_{x}=-\bar{\kappa}_{y}= \pm \sqrt{-\frac{(1-\nu)}{\phi}-\bar{g}_{\mathrm{A}}}
$$

Again, there are two sets of shapes but this time, their stability depends on the shear modulus, $\alpha$; in particular, upon the eigenvalue associated with twist - the final element in the stability matrix, which can be shown to be equal to $4 \alpha-2 \phi\left(\bar{\kappa}_{x} \bar{\kappa}_{y}-\bar{g}_{\mathrm{A}}\right)$. After substituting for $\bar{\kappa}_{x}$ and $\bar{\kappa}_{y}$ above, this term is positive when $\alpha>(1-\nu) / 2$, and the above are valid bistable shapes. Otherwise, it is zero for material isotropy, indicating that there is no stiffness in this direction: the disk is neutrally stable in a torsional manner with principal shapes according to the above expressions. We may think of any intermediate shapes being described by the above but where the coordinate axes have been rotated by some nominal angle about a vertical axes. Recall that we are not considering $\alpha<(1-\nu) / 2$ suffice to say that all solutions would be unstable in this case - a different solution regime governs.

Figure 1 compares these predictions to a finite element analysis using the software package ABAQUS (Hibbitt et al. 2011). The disk is circular and comprises triangular shell elements, and a small initial out-ofplane displacement is imposed upon the flat shape, in order to seed the potential for buckling. The growth strain is specified using an inelastic strain field, $\epsilon_{\mathrm{A}}=\epsilon_{\mathrm{A}}(x, y)$, with the elementary distribution

$$
\epsilon_{\mathrm{A}}(x, y)=-\bar{\epsilon} \frac{\left(x^{2}+y^{2}\right)}{a^{2}} \Rightarrow \bar{g}_{\mathrm{A}}=4 \bar{\epsilon} \frac{a^{2}}{t^{2}}
$$

using Eqn 3. The maximum value, $\bar{\epsilon}$, would be defined by the growth or actuation strain capabilities of the actual material, and we use it here to control the degree of positive and negative growth by increasing or decreasing its value from zero, respectively. A geometrically non-linear, large-displacement analysis incorporating a linearly elastic, isotropic material response is specified, and the disk is centrally fixed against rigid body movement, being free from externally applied forces and moments everywhere else. No restrictions are imposed upon the expected profile of curvatures, and Fig. 1 records the highest displacements availed by the edge of the disk. The discrepancy between theory and computation diverges slightly as growth proceeds but the trends are encouragingly similar despite the ratio of displacement to disk thickness being well above unity - the notional limit of shallow displacements, and despite our assumption of linearity via Eqn 1: for non-shallow displacements overall, the agreement is unexpectedly good. Additional pictures of the postbuckled shape indicate that there is constant Gaussian curvature throughout most of the disk except for a boundary layer on the periphery, where the internal bending moments fall away to zero non-uniformly in accordance with an exact solution. Thus, our assumption of uniform shape is not valid close to the edge 
but this local absence of correctness has minimal influence on the edge displacements because of the small width of the boundary layer. Note that it is difficult to see the equivalent strain at buckling owing to the large range of growth overall.

(b) Bending growth, $\bar{g}_{\mathrm{A}}=0,\left(\bar{\kappa}_{x \mathrm{~A}}, \bar{\kappa}_{y \mathrm{~A}}\right) \neq 0$

Recall that $\bar{\kappa}_{y \mathrm{~A}}=+\bar{\kappa}_{y \mathrm{~A}}$ or $\bar{\kappa}_{y \mathrm{~A}}=-\bar{\kappa}_{x \mathrm{~A}}$ for Eqns 11 and 13 to apply and, in order to distinguish formally this application, we denote the former as a case of equal-sense growth and the latter as opposite-sense: in the previous case of $\bar{g}_{\mathrm{A}} \neq 0$, there is no commensurate distinction in the governing equations although we may think of similar behaviour depending on the sign of $\bar{g}_{\mathrm{A}}$. Each of these cases is now treated separately for the sake of clarity and completeness - the solution curvatures are non-trivial expressions, and they are also similar to the solutions found by (Seffen and Guest 2011), as noted before.

\section{(b.i) Equal-sense bending growth}

The disk curvatures are the same with respect to using Eqn 13 but different in Eqn 11 and, since the growth strains are always the same $\left(\bar{\kappa}_{x \mathrm{~A}}=\bar{\kappa}_{y \mathrm{~A}}\right)$, it is reasonable to expect that the disk deflects with equal curvatures initially as the growth increases from zero. Thus, Eqn 13 is considered first with the term $\bar{g}_{\mathrm{A}}=0$. The final form and conditions for its application may be written as:

$$
\bar{\kappa}_{y \mathrm{~A}}=\bar{\kappa}_{x \mathrm{~A}}: \bar{\kappa}_{y}=\bar{\kappa}_{x} ; \quad 0 \leq \bar{\kappa}_{x \mathrm{~A}}<\bar{\kappa}_{x \mathrm{~A}}^{*} \Rightarrow \bar{\kappa}_{x}+\frac{\phi}{(1+\nu)} \bar{\kappa}_{x}^{3}=\bar{\kappa}_{x \mathrm{~A}}
$$

It is possible to solve for $\bar{\kappa}_{x}$ explicitly in terms of $\bar{\kappa}_{x \mathrm{~A}}$ but this is not a compact expression and, therefore, is not presented. The growth limit for this mode is defined as $\bar{\kappa}_{x \mathrm{~A}}^{*}$, which is quantified momentarily, and is the limit of stable behaviour when considering the eigenvalues of Eqn 14. Thereafter, the disk curvatures cannot be the same, and Eqn 11 now governs. It can be solved explicitly under the following conditions to reveal the exact disk curvatures as:

$$
\begin{gathered}
\mu=1, \quad \bar{\kappa}_{y \mathrm{~A}}=\bar{\kappa}_{x \mathrm{~A}}: \bar{\kappa}_{y} \neq \bar{\kappa}_{x}, \quad \bar{\kappa}_{x \mathrm{~A}}>\bar{\kappa}_{x \mathrm{~A}}^{*} \Rightarrow \phi \bar{\kappa}_{x}^{2}-\phi(1+\nu) \bar{\kappa}_{x} \bar{\kappa}_{x \mathrm{~A}}+(1-\nu)=0 \\
\Rightarrow \quad \bar{\kappa}_{x}=\frac{\bar{\kappa}_{x \mathrm{~A}}(1+\nu)}{2}\left[1 \pm\left[1-\left(\frac{\bar{\kappa}_{x \mathrm{~A}}^{*}}{\bar{\kappa}_{x \mathrm{~A}}}\right)^{2}\right]^{0.5}\right], \quad \bar{\kappa}_{y}=\frac{\bar{\kappa}_{x \mathrm{~A}}(1+\nu)}{2}\left[1 \mp\left[1-\left(\frac{\bar{\kappa}_{x \mathrm{~A}}^{*}}{\bar{\kappa}_{x \mathrm{~A}}}\right)^{2}\right]^{0.5}\right]
\end{gathered}
$$

The term $\bar{\kappa}_{x \mathrm{~A}}^{*}$ (and $\bar{\kappa}_{y \mathrm{~A}}^{*}$ ) defines the growth strain at which the real roots of the governing equation become available and is found to be equal to $\sqrt{4(1-\nu) / \phi(1+\nu)^{2}}$. A formal stability investigation also confirms that there is a switch from one stable solution to the other at $\bar{\kappa}_{x \mathrm{~A}}^{*}$.

Compared to the effects of $\bar{g}_{\mathrm{A}}$, the disk distorts out-of-plane with equal curvatures from the outset as it attempts to reach $\bar{\kappa}_{x \mathrm{~A}}$ and $\bar{\kappa}_{y \mathrm{~A}}$ under Eqn 19. It then buckles when the imposed Gaussian curvature reaches a value of $\bar{\kappa}_{x \mathrm{~A}}^{*} \bar{\kappa}_{y \mathrm{~A}}^{*}$. At this point, the Gaussian curvature of the disk is $\bar{\kappa}_{x} \bar{\kappa}_{y}=(1-\nu) / \phi$, and this remains fixed during post-buckling since $\mu=1$ irrespective of the value of $\bar{\kappa}_{x \mathrm{~A}}$ and $\bar{\kappa}_{y \mathrm{~A}}$. The individual curvatures, $\bar{\kappa}_{x}$ and $\bar{\kappa}_{y}$, increase or decrease according to their closed-form solutions in Eqn 20 as growth increases, to form an open cylindrical shape in the limit: but their product remains conserved and the post-buckling growth mode is almost developable. Furthermore, when $\alpha>(1-\nu) / 2$, it also has two stable configurations, depending on the internal signs of Eqn 20. For material isotropy, post-buckling is neutrally stable and the disk can swap shapes without a change in the stored energy; when $\alpha<(1-\nu) / 2$, a different regime of stable twisted shapes replaces the current set, but these are not being considered as noted before.

The maximum and minimum displacements on the edge of the disk are compared in Fig. 2 for both theory and a finite element analysis of the same computational scheme as before, but where the imposed curvatures are specified by another strain field, $\epsilon_{\mathrm{A}}=\epsilon_{\mathrm{A}}(z)$, which only varies through the thickness, and linearly, such that (dimensional) 


$$
\epsilon_{\mathrm{A}}(z)=\frac{2 z \bar{\epsilon}}{t} \Rightarrow \kappa_{x \mathrm{~A}}=\kappa_{y \mathrm{~A}}=\frac{2 \bar{\epsilon}}{t}
$$

where, again, $\bar{\epsilon}$ is a maximum material strain.

At buckling, there is a clear divergence, or bifurcation, in displacements, with rapid and disparate changes in values over a short increment in growth strains. The computation also captures this feature and, as with extensional growth, the overall trends are exceptionally close even for large displacements. It is also clear that the larger curvature tends towards a linear variation with increasing growth strain and, again, the shapes of disk closely match the assumption of uniformity.

(b.ii) Opposite-sense bending growth

When $\bar{\kappa}_{x \mathrm{~A}}=-\bar{\kappa}_{y \mathrm{~A}}$, it may be verified that the shape of the disk is defined by the following solution sets:

$$
\bar{\kappa}_{y \mathrm{~A}}=-\bar{\kappa}_{x \mathrm{~A}}: \bar{\kappa}_{y}=-\bar{\kappa}_{x} ; \quad 0 \leq \bar{\kappa}_{x \mathrm{~A}}<\bar{\kappa}_{x \mathrm{~A}}^{*} \Rightarrow \bar{\kappa}_{x}+\frac{\phi}{(1-\nu)} \bar{\kappa}_{x}^{3}=\bar{\kappa}_{x \mathrm{~A}}
$$

and

$$
\begin{array}{r}
\bar{\kappa}_{y \mathrm{~A}}=-\bar{\kappa}_{x \mathrm{~A}}: \bar{\kappa}_{y} \neq \bar{\kappa}_{x}, \mu=-1 ; \bar{\kappa}_{x \mathrm{~A}}>\bar{\kappa}_{x \mathrm{~A}}^{*} \Rightarrow \phi \bar{\kappa}_{x}^{2}-\phi(1-\nu) \bar{\kappa}_{x} \bar{\kappa}_{x \mathrm{~A}}+(1+\nu)=0 \\
\Rightarrow \bar{\kappa}_{x}=\frac{\bar{\kappa}_{x \mathrm{~A}}(1-\nu)}{2}\left[1 \pm\left[1-\left(\frac{\bar{\kappa}_{x \mathrm{~A}}^{*}}{\bar{\kappa}_{x \mathrm{~A}}}\right)^{2}\right]^{0.5}\right], \bar{\kappa}_{y}=-\frac{\bar{\kappa}_{x \mathrm{~A}}(1-\nu)}{2}\left[1 \mp\left[1-\left(\frac{\bar{\kappa}_{x \mathrm{~A}}^{*}}{\bar{\kappa}_{x \mathrm{~A}}}\right)^{2}\right]^{0.5}\right]
\end{array}
$$

with $\bar{\kappa}_{x \mathrm{~A}}^{*}\left(=-\bar{\kappa}_{y \mathrm{~A}}^{*}\right)$ equal to $\sqrt{4(1+\nu) / \phi(1-\nu)^{2}}$. Initially, the disk deflects into a saddle shape before buckling into an almost developable mode at $\bar{\kappa}_{x \mathrm{~A}}^{*}$. As with the previous case, the Gaussian curvature is conserved in this mode but post-buckling is always bistable. A finite element analysis was not performed for the sake of brevity.

\section{(c) Summary and comparison of modes}

Both growth modes attempt to induce a change in the Gaussian curvature of the disk, which can be prescribed as a positive or negative change. The disk responds differently but buckling is common to both modes: during extensional growth, buckling precedes an uptake of Gaussian curvature by the disk whereas buckling during bending growth signifies that the disk has acquired maximum Gaussian curvature. If our descriptor of shape-change is taken to be some measure of the current Gaussian curvature, then extensional growth always dominates bending, at least beyond buckling. But if we are interested in maximising displacements - if, for example as engineers, we are designing an actuator of shape, Gaussian curvature is no longer important; rather, it is the performance of a single curvature because displacements are largest on the edge and given by $\bar{\kappa} t / 2$ under the assumption of uniformity. The previous closed-form curvature expressions provide some compact insight when there are large, post-buckled growth strains, when $\bar{g}_{\mathrm{A}}>>\bar{g}_{\mathrm{A}}^{*}$ and $\bar{\kappa}_{x \mathrm{~A}}>>\bar{\kappa}_{x \mathrm{~A}}^{*}$. For positive growth strains, the asymptotic limits describing one of the bistable shapes can be shown to be:

$$
\begin{gathered}
\text { extensional : } \quad \bar{\kappa}_{x} \bar{\kappa}_{y} \rightarrow \bar{g}_{\mathrm{A}} ; \quad \bar{\kappa}_{x} / \sqrt{\bar{g}_{\mathrm{A}}} \rightarrow 1, \quad \bar{\kappa}_{y} / \sqrt{\bar{g}_{\mathrm{A}}} \rightarrow 1 \\
\text { bending : } \quad \bar{\kappa}_{x} \bar{\kappa}_{y}=(1-\nu) / \phi, \quad \bar{\kappa}_{x} / \bar{\kappa}_{x \mathrm{~A}} \rightarrow 1+\nu, \quad \bar{\kappa}_{y} / \bar{\kappa}_{x \mathrm{~A}} \rightarrow 0
\end{gathered}
$$

As noted before, the Gaussian curvature arising from bending growth does not depend on $\bar{\kappa}_{x \mathrm{~A}}$ but has a constant finite value: each of its constituent curvatures tends towards a very large number and zero but their product is always fixed and independent of $\bar{\kappa}_{x \mathrm{~A}}$. For the other bistable mode, $\bar{\kappa}_{x}$ and $\bar{\kappa}_{y}$ simply swap values.

When there are negative growth strains, then: 


$$
\begin{gathered}
\text { extensional : } \quad \bar{\kappa}_{x} \bar{\kappa}_{y} \rightarrow-\bar{g}_{\mathrm{A}} ; \quad \bar{\kappa}_{x} / \sqrt{\bar{g}_{\mathrm{A}}} \rightarrow 1, \quad \bar{\kappa}_{y} / \sqrt{\bar{g}_{\mathrm{A}}} \rightarrow-1 \\
\text { bending : } \quad \bar{\kappa}_{x} \bar{\kappa}_{y}=-(1+\nu) / \phi, \quad \bar{\kappa}_{x} / \bar{\kappa}_{x \mathrm{~A}} \rightarrow 1-\nu, \quad \bar{\kappa}_{y} / \bar{\kappa}_{x \mathrm{~A}} \rightarrow 0
\end{gathered}
$$

In either set of expressions, the curvatures are not correlated across modes unless the growth strains are related to each other, and usefully so. In the above, there is little difference between the largest displacements if we assume that $\sqrt{\bar{g}_{\mathrm{A}}}$ is the same size as $\bar{\kappa}_{x \mathrm{~A}}$, but this is an arbitrary statement. Furthermore, the above limits are simply expressed but unlikely to be reached in practice - at least, for engineering materials, where actuation strains are typically no more than 1\% (Huber et al. 1997), and understanding the performance at lower growth strains close to, and around, buckling is imperative.

The most straightforward scheme is led by the finite element approach, in which the distributions of growth strains are the simplest they can be so that $G\left(\epsilon_{\mathrm{A}}\right)$ and $\operatorname{det} \kappa_{\mathrm{A}}$ from Section 2 are constant but which are now tied together by the fundamental material limit, $\bar{\epsilon}$. Equations 18 and 21 are employed but with a slight modification to the bending growth strain so that both equal- and opposite-sense growth are specified. The final dimensionless expressions read as:

$$
\bar{g}_{\mathrm{A}}=4 \bar{\epsilon} \frac{a^{2}}{t^{2}} ; \quad \bar{\kappa}_{x \mathrm{~A}}=\gamma|\bar{\epsilon}| \frac{a^{2}}{t^{2}}, \quad \bar{\kappa}_{y \mathrm{~A}}=\gamma \overline{\bar{\epsilon}} \frac{a^{2}}{t^{2}}
$$

The sign of $\bar{\epsilon}$ determines when there is positive or negative extensional growth, as well as the sign of $\bar{\kappa}_{y \mathrm{~A}}$ : when $\bar{\epsilon}>0$, the sign is positive and there is equal-sense growth, and vice versa; the absolute sign in $\bar{\kappa}_{x \mathrm{~A}}$ above ensures that it always takes a positive value. The constant term, $\gamma$, accounts for the effectiveness of bending relative to the maximum growth strain, and has an upper value of two when the strains are equal to $\pm \bar{\epsilon}$ on the disk upper and lower surfaces, which is the absolute range in view of the limit of linear elastic behaviour. When $\gamma$ is less than two, then bending growth strains are not optimally distributed through the disk thickness, as might arise when not all of the material is capable of furnishing growth strains.

Notwithstanding, a comparison of the largest displacements between growth modes is shown in Fig. 3 after substituting $\bar{g}_{\mathrm{A}}$ and $\bar{\kappa}_{x \mathrm{~A}}$ back into the specific governing equations. On the abscissa, we retain the control parameter, $\bar{\epsilon}(a / t)^{2}$, so that geometrical, as well as material properties can be compared, and on the ordinate, the ratio of maximum displacements, $d_{\mathrm{S}} / d_{\mathrm{B}}$, where superscripts $\mathrm{S}$ and $\mathrm{B}$ refer to extension and bending, respectively. We also consider positive and negative growth, and specify three values of $\gamma$ equal to $1 / 3,1$ and 2 .

Initially, each displacement ratio is zero and then rises steeply after buckling. It is larger than unity when $\gamma$ is smaller and closer to zero and, more so, when growth is negative. The discontinuity of slope and subsequent downturn in the ratio are due to the sharp increase in one of the bending curvatures after bifurcating. Thereafter, the trend is generally downwards since the largest bending displacement approximately varies with growth in a linear fashion, as displayed in Fig. 2, whilst the extensional displacements grow in accordance with $\sqrt{\bar{\epsilon}}$. Conditions on the ratio cross-over can be calculated if desired, but Fig. 3 testifies that generally, extensional displacements are higher only when $\bar{\epsilon}(a / t)^{2}$ is much less than 100 - when disks tend to be thick or when growth strains tend to be small. Also plotted are the asymptotic displacement ratios for each value of $\gamma$, which can be verified as being $2 t /(\gamma(1 \pm \nu) \sqrt{\mid \bar{\epsilon}} a)$. These approximations very quickly match predictions and may be used as an informative measure of performance.

There are of course, other ways to compare shape performance without focussing on displacements, for example, by comparing the strain energy stored in the disk, and one such case is reported later. Beforehand, we consider the complementary effects of both growth modes.

\section{Double Mode Growth}

The obvious differences in shape performance between the previous single modes of growth suggest a possible richness of behaviour when they act together. Along with the variation in shape, there is the degree of stability 
and its connection to buckling; specifically, that bistable or neutrally stable configurations are wrought during post-buckling. Analytically, the governing equations of deformation produce two real roots, which happen to be stable. Given the algebraic nature of solution, it is possible to establish regions of interest for multistable behaviour from the properties alone of the specific governing equations, Eqns 11 and 13. In this regard, the discriminant of each of the polynomial expressions is key (Seffen 2007) for when the discriminant is negative, there are at least two real roots and thus, potentially, bistable behaviour. The boundaries between regions of different stabilities are therefore established by setting the discriminants equal to zero and plotting the corresponding loci in "growth" space, $\left(\bar{g}_{\mathrm{A}}, \bar{\kappa}_{\mathrm{A}}\right)$, before assessing stability, which is confirmed separately by solving explicitly for some - not all-equilibria within the regions of interest and testing their eigenvalue performance using Eqn 14. Recall that we only consider the shear modulus, $\alpha$, to be isotropic or larger in value.

Consider, first, the full governing equations for equal-sense bending growth $\left(\bar{\kappa}_{x \mathrm{~A}}=\bar{\kappa}_{y \mathrm{~A}}\right)$, which are repeated below with $\beta=1$, in order to underline their discriminant expressions:

$$
\phi \bar{\kappa}_{x}^{3}+\left[(1+\nu)-\phi \bar{g}_{\mathrm{A}}\right] \bar{\kappa}_{x}-\bar{\kappa}_{x \mathrm{~A}}(1+\nu)=0 ; \phi \bar{\kappa}_{x}^{2}-\phi \bar{\kappa}_{x} \bar{\kappa}_{x \mathrm{~A}}(1+\nu)+(1-\nu)+\phi \bar{g}_{\mathrm{A}}=0
$$

The discriminants are found straightforwardly (Weisstein 2006) and are denoted as $d_{1}$ and $d_{2}$ :

$$
4\left[\phi \bar{g}_{\mathrm{A}}-(1+\nu)\right]^{3}-27 \phi \bar{\kappa}_{x \mathrm{~A}}^{2}(1+\nu)^{2}=d_{1} ; \quad \phi^{2} \bar{\kappa}_{x \mathrm{~A}}^{2}(1+\nu)^{2}-4 \phi\left[(1-\nu)+\phi \bar{g}_{\mathrm{A}}\right]=d_{2}
$$

These are then set equal to zero and plotted in Fig. 4 with coordinates $\left(\bar{g}_{\mathrm{A}}, \bar{\kappa}_{x \mathrm{~A}}\right)$, where $\bar{\kappa}_{x \mathrm{~A}}=\bar{\kappa}_{y \mathrm{~A}}$ in the first two quadrants. It is then straightforward to verify that those coordinate points which lie above the locus of $d_{1}=0$ as drawn ensure that this discriminant is negative, and that this region has possibly more than one stable equilibria. The corresponding behaviour for the second discriminant has any point lying below the curve of $d_{2}=0$. In fact, the former is a bistable region only when $\alpha$ is larger than $(1-\nu) / 2$, otherwise, it refers to neutrally stable disks when isotropy is upheld: the latter region always conforms to bistability. Interestingly, the loci can cross over in the upper portion of the first quadrant so that each bistable region overlaps. This produces a region of tristable shapes, and this overlapping, in physical terms, can be thought of as two sets of bistable shapes "mixing" but where two of the equilibria - originally very similar in shapehave now converged into a single stable shape; the other two shapes are sufficiently different to remain distinct. For isotropic disks, stability is lost in this region because the lower bistable region overlaps the neutrally stable region, and the disk equilibria are a mixture of these modes. Finally, there is a monostable region, which encloses the origin, where the discriminants, $d_{1}$ and $d_{2}$ are greater than zero.

For opposite-sense bending growth $\left(\bar{\kappa}_{x \mathrm{~A}}=-\bar{\kappa}_{y \mathrm{~A}}\right)$, the corresponding discriminants of the specific governing equations may be verified as:

$$
4\left[-\phi \bar{g}_{\mathrm{A}}-(1-\nu)\right]^{3}-27 \phi \bar{\kappa}_{x \mathrm{~A}}^{2}(1-\nu)^{2}=d_{3} ; \quad \phi^{2} \bar{\kappa}_{x \mathrm{~A}}^{2}(1-\nu)^{2}-4 \phi\left[(1+\nu)-\phi \bar{g}_{\mathrm{A}}\right]=d_{4}
$$

The third and fourth quadrants in Fig. 4 are now created by plotting the ordinate, $\bar{\kappa}_{x \mathrm{~A}}\left(=-\bar{\kappa}_{y \mathrm{~A}}\right)$, downwards. The stability complexion is, however, very different because one of the roots afforded by $d_{3}$ being negative is never stable, as confirmed separately. Only $d_{4}<0$ produces bistable solutions, which are not affected by the shear modulus and, hence, opposite-sense bending growth is simply divided into two regions by the curve, $d_{4}=0$, as shown. There is an additional horizontal boundary extending outwards from $\bar{g}_{\mathrm{A}}^{*}=-(1-\nu) / \phi$ along the $\bar{g}_{\mathrm{A}}$ axis, in order to distinguish the bistability in the equal-sense region above from monostability beneath.

In the complete picture, each region can be labelled as follows: I, monostable; II, bistable when $\alpha>$ $(1-\nu) / 2$ otherwise neutrally stable; III, bistable; IV, tristable for orthotropy or mono- and neutrally stable for isotropy. Equal- and opposite-sense bending growth regions are demarcated by extra labels of "a" and "b", and the four intercepts on the axes, labelled A-D, confirm the four single-mode buckling values $\left(\kappa_{x \mathrm{~A}}^{*}, g_{\mathrm{A}}^{*}\right)$ from Section 4. Some sense of the shapes of disk in each region are given in Fig. 5.

The corresponding degree of shape-change in the disk can be tackled in several ways but we take our lead from Fig. 3 by wishing to incorporate the influence of the fundamental material strain limit, $\bar{\epsilon}$. This is 
important for actuator design but the corresponding definitions of $\bar{g}_{\mathrm{A}}$ and $\bar{\kappa}_{x \mathrm{~A}}$ in Eqn 24 are tied by $\bar{\epsilon}$, so we need to introduce an additional parameter, in order to separate their effects meaningfully. This is achieved most simply by specifying a weighted sum of each contribution in Eqn 24:

$$
\bar{g}_{\mathrm{A}}=(1-|\eta|) 4 \bar{\epsilon} \frac{a^{2}}{t^{2}} ; \quad \bar{\kappa}_{x \mathrm{~A}}=|\eta \bar{\epsilon}| \frac{a^{2}}{t^{2}}, \quad \bar{\kappa}_{y \mathrm{~A}}=\eta|\bar{\epsilon}| \frac{a^{2}}{t^{2}}
$$

The parameter, $\eta$, ranges from -1 to 1 such that when $\eta=0$, there can be only extensional growth; when $\eta= \pm 1$, there is only bending growth but the sign of $\eta$ determines the sign of $\bar{\kappa}_{y \mathrm{~A}}$, which is equal in magnitude to $\bar{\kappa}_{x \mathrm{~A}}$ : for $\eta>0$, there is equal-sense bending growth, and for $\eta<0$, opposite-sense behaviour. The sign of $\bar{\epsilon}$ only determines whether there is positive or negative extensional growth and does not affect the direction of bending growth. Note that $\gamma$ is set equal to unity in the above compared to Eqn 24, merely to set the maximum strain range anywhere in the disk equal to $\bar{\epsilon}$.

Figure 6 indicates the maximum possible displacement of the disk under these conditions, which is plotted as a series of contours of the ratio of displacement to disk thickness. It is clear that these are largest when confined to corners of the plot — when $\bar{\epsilon}$ is high and when bending growth dominates - and the abrupt changes in the direction of contours arises when there is a change in the responsible equilibrium shape of largest displacement. The strain energy stored in the disk for each responsible equilibrium shape in Fig. 6 is then calculated via Eqn 6 and plotted in Fig. 7 with the same coordinate axes. The landscape rises as we move towards the corners, suggesting that the disk acquires more strain energy during bending growth, but otherwise, it is generally featureless except, to note, that it dips towards zero in places. Careful examination shows that, in fact, these are exactly zero-value contours, which are indicated as the pair of dotted lines in the first and third quadrants. By overlaying the same lines onto Fig. 6, we see that the corresponding maximum displacement along them is non-zero, which presents a peculiarity: that some growth-induced displacements are permissible but store no strain energy. This is resolved in the following section as we note that the continuous nature of these zero-energy contours must indicate some special relationship between extensional and bending growth modes.

\section{$6 \quad$ Natural Growth}

The specific governing equation, Eqn 13, can be factorised when $\bar{g}_{\mathrm{A}}$ is set equal to $\bar{\kappa}_{x \mathrm{~A}}^{2}\left(\bar{\kappa}_{y}=\bar{\kappa}_{x}\right)$ or $-\bar{\kappa}_{x \mathrm{~A}}^{2}$ $\left(\bar{\kappa}_{y}=-\bar{\kappa}_{x}\right)$, leading to the solution set $\bar{\kappa}_{x}=\bar{\kappa}_{x \mathrm{~A}}= \pm \bar{\kappa}_{y}$, depending on the choice of sign. In these cases, $\mu=\nu$ follows, and the eigenvalues of the stability matrix can be verified as being $1 \pm \nu+\phi \bar{\kappa}_{x \mathrm{~A}}^{2}$ and $4 \alpha$, which are always positive and give rise to a stable performance in either case. At the same time, this factorisation produces no strain energy in the disk when the solution curvatures and growth strains are substituted back into Eqn 6 . Interestingly, as $\bar{g}_{\mathrm{A}}\left(\right.$ and $\left.\bar{\kappa}_{x \mathrm{~A}}\right)$ increase, or decrease, there is no increase in $\bar{U}$ as growth proceeds; but the positive eigenvalues stipulate that any perturbation away from these equilibrium states always produces a restorative rise in strain energy. This is quite different from the neutral stability reported earlier, where the disk is stiff in the direction of the imposed growth strains but has zero torsional stiffness orthogonal to them. Here, the structure is stiff in the sense of departures from the growth mode, but it has effectively zero stiffness in the sense or growth proceeding. This is similar in performance to a recently-discovered class of pin-jointed, tensegrity structures, where special pre-stressing elements allows for joint displacements without energy penalty (Schenk et al. 2007).

The fundamental reason for this behaviour can be gleaned from thinking about the conditions of shape alone. Recall from Section 2 that the current shape must obey the compatibility requirement, $\operatorname{det} \kappa=G(\epsilon)$, but that the imposed strains are not obliged to; however, it is clear that $\operatorname{det} \kappa_{\mathrm{A}}=G\left(\epsilon_{\mathrm{A}}\right)=g_{\mathrm{A}}$ in the present case, and that $\operatorname{det} \kappa=\operatorname{det} \kappa_{\mathrm{A}}$. In other words, both compatibility equations are satisfied, thereby precluding the self-stresses that lead to a build-up of strain energy. The coupled growth mode here is a natural mode since the disk exactly follows the target shape. In an overall sense, energy must be invested in the disk so that $\bar{\kappa}_{x \mathrm{~A}}$ and $\bar{g}_{\mathrm{A}}$ are wrought in the first place, but there is no extra component due to elastic stresses, and 
the complete performance of the system is limited only by the thermodynamical capabilities of practical growth and actuation processes.

\section{Conclusions}

The distinct topics of growth and of actuation of shape in thin shells have been treated together and compared in this paper. We have distinguished between them in a simple way, by assuming that they confer, respectively, extensional and bending strains upon an initially flat disk, and we have considered this as our "growth" scenario of two growth modes, but which can be applied to both natural and artificial systems. The distribution of strains have been deliberately selected so that growth ultimately imposes uniform changes in the Gaussian curvature. The disk responds elastically by trying to match the change insofar as compatibility demands but generally lags behind it because of the build-up in stored strain energy. Both growth modes produce different characteristics of shape but buckling is an inherent property: bending growth buckles into an almost developable mode of constant Gaussian curvature whereas extensional growth increases the Gaussian curvature only after buckling. The displacement performance is different because it is controlled by the largest curvature, not the Gaussian, and we have shown that extensional growth produces larger displacements when the product of maximum material growth strain, $\bar{\epsilon}$, and $(a / t)^{2}$ is typically less than 100 and usually less than 25 , and vice-versa. In general, more than one post-buckling equilibrium configuration can exist for given levels of growth, either singly or in combination and, in practice, the disk grows into one of these shapes and only "moves" into the others under externally applied forces. There are neutrally stable shapes in which the disk has no torsional stiffness although it remains stiff in bending, otherwise, bistability, and sometimes tristability, prevails in the post-buckling regimes. Where possible, closed-form expressions of shape have been determined, and a convenient map of regimes with well-defined boundaries has been used to convey the overall stability, displacement- and strain energy performances. Finally, we note that natural growth is possible when the shape of the disk exactly matches the imposed growth strains, but only when the latter are related by the condition, $\bar{g}_{\mathrm{A}}= \pm \bar{\kappa}_{x \mathrm{~A}}^{2}$ : it is possible to create both positive and negative Gaussian curvature from a flat disk without storing any extensional and bending strain energy.

\section{Acknowledgments}

The authors were able in to discuss their findings in person through the generous support of the Royal Society (KAS) and CNRS (CM). The authors are also very grateful to the two anonymous referees for useful comments during review.

\section{References}

L Aouar, Y Chebli, and A Geitmann. Morphogenesis of complex plant cell shapes - the mechanical role of crystalline cellulose in growing pollen tubes. Sexual Plant Reproduction, 23:15-27, 2010.

S Armon, E Efrati, R Kupferman, and E Sharon. Geometry and mechanics in the opening of chiral seed pods. Science, 333:1726-1729, 2011.

C R Calladine. Theory of Shell Structures. Cambridge University Press, 1983.

O Campas and L Mahedvan. Shape and dynamics of tip-growing cells. Current Biology, 19:2102-2107, December 2009.

E Cerda and L Mahadevan. Geometry and physics of wrinkling. Physical Review Letters, 90(7):074302-1 to 074302-4, February 2003. 
$\mathrm{X}$ Chen and J Yin. Buckling patterns of thin films on curved compliant substrates with applications to morphogenesis and three-dimensional micro-fabrication. Soft Matter, 6:5667-5680, 2010.

J Dervaux and M Ben-Amar. Morphogenesis of growing soft tissues. Physical Review Letters, pages 068101-1 to $068101-4$, August 2008.

J Dervaux, P Ciarletta, and M Ben Amar. Morphogenesis of thin hyperleastic plates: a constitutive theory of biological growth in the Foppl-von Karman limit. Journal of the Mechanics and Physics of Solids, 57: 458-471, 2009.

J Dumais and C R Steele. New evidence for the role of mechanical forces in the shoot apical meristem. Journal of Plant Growth Regulation, 19:7-18, 2000.

A Fernandes, C Maurini, and S Vidoli. Multiparameter actuation for shape control of bistable composite plates. International Journal of Solids and Structures, 47(10):1449-1458, 2010.

Y Forterre and J Dumais. Generating helices in Nature. Science, 333:1715-1716, 2011.

Y Forterre, J M Skotheim, J Dumais, and L Mahadevan. How the Venus Flytrap snaps. Nature, 433:421-425, January 2005.

L B Freund. Substrate curvature due to thin film mismatch strain in the nonlinear deformation range. Journal of the Mechanics and Physics of Solids, 48:1159-1174, 2000.

A Goriely and M Ben Amar. Differential growth and instability in elastic shells. Physical Review Letters, pages $198103-1$ to $198103-4$, May 2005.

S D Guest and S Pellegrino. Analytical models for bistable cylindrical shells. Proceedings of the Royal Society, London, A, 462:839-854, 2006.

Hibbitt, Karlsson, and Sorenson. ABAQUS Version 6.9. Hibbitt, Karlsson \& Sorenson, Inc., Pawtucket, 2011.

J E Huber, N A Fleck, and M F Ashby. The selection of mechanical actuators based on performance indices. Proceedings of the Royal society, London, A, 453:2185-2205, 1997.

Y Klein, E Efrati, and E Sharon. Shaping of elastic sheets by prescription of non-Euclidean metrics. Science, 315:1116-1120, February 2007.

M A R Koehl, W K Silk, H Liang, and L Mahadevan. How kelp produce blade shapes suited to different flow regimes: a new wrinkle. Integrative and Comparative Biology, 48(6):834-851, 2008.

S Kyriakides. Propagating instabilities in structures. In J W Hutchinson and T Y Wu, editors, Advances in Applied Mechanics, pages 67-189. Academic Press, 1994.

H Liang and L Mahadevan. The shape of a long leaf. Proceedings of the National Academy of Sciences, 106 (52):22049-22054, 2009.

C D Modes, K Battacharya, and M Warner. Gaussian curvature from flat elastica sheets. Proceedings of the Royal Society, London, A, 467:1121-1140, 2011.

U Nath, B C W Crawford, R Carpenter, and E Coen. Genetic control of surface curvature. Science, 299: 1404-1407, February 2003.

M Schenk, S D Guest, and J L Herder. Zero stiffness tensegrity structures. International Journal of Solids and Structures, 44(20):6569-6583, 2007. 
K A Seffen. Morphing bistable orthotropic elliptical shallow shells. Proceedings of the Royal Society, London, A, 463(2077):67-83, 2007.

K A Seffen and S D Guest. Prestressed morphing bistable and neutrally stable shells. Journal of Applied Mechanics, Transactions ASME, 78(1):011002-1 to 011002-6, 2011.

K A Seffen and R A McMahon. Thermal buckling of a uniform disk. International Journal of Mechanical Sciences, 49(2):230-238, 2007.

S Vidoli and C Maurini. Tristability of thin orthotropic shells with uniform initial curvature. Proceedings of the Royal Society of London, A, 464:2949-2966, 2008.

E W Weisstein. Polynomial discriminant. Wolfram Web Resource http://mathworld.wolfram.com/, 2006.

W H Wittrick, D M Myers, and W R Blunden. Stability of a bimetallic disk. Quarterly Journal of Mechanics and Applied Mathematics, 6(1):15, 1953. 


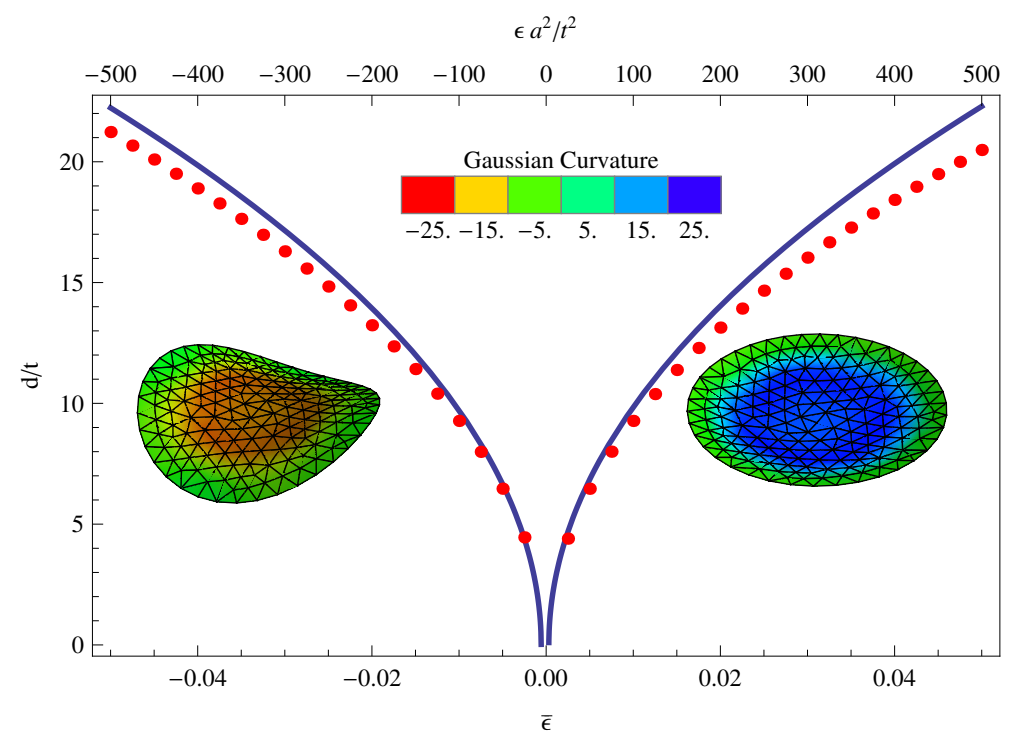

Figure 1: Comparison of the largest out-of-plane displacements, $d$, on the edge of a circular disk subjected to extensional growth. The disk has radius $a=0.1 \mathrm{~m}$, thickness $t=0.001 \mathrm{~m}$, and the material is isotropic with Poisson's ratio $\nu=0.3$. The parameter, $\bar{\epsilon}$, is the maximum growth strain given by Eqn 18, and is a growth control parameter: when $\bar{\epsilon}>0$, the disk grows into a cap of positive Gaussian curvature with all edge points at maximum displacement; $\bar{\epsilon}<0$ produces a saddle shape with only two points maximally displaced upwards and downwards; close to the origin, there is no displacement before buckling at $\bar{\epsilon} \approx 0.6 \times 10^{-3}$. Solid lines are theoretical predictions from Section 2 and dots are from a non-linear finite element analysis (FEA). The indicated shapes are also taken from the FEA after the disk buckles for growth in either direction. The legend shows the degree of Gaussian curvature in both cases, and over the bulk of the disk it is constant, which affirms the assumption of uniform curvatures. Near the periphery, it attenuates to zero because the real boundary condition enforces zero moment there. 


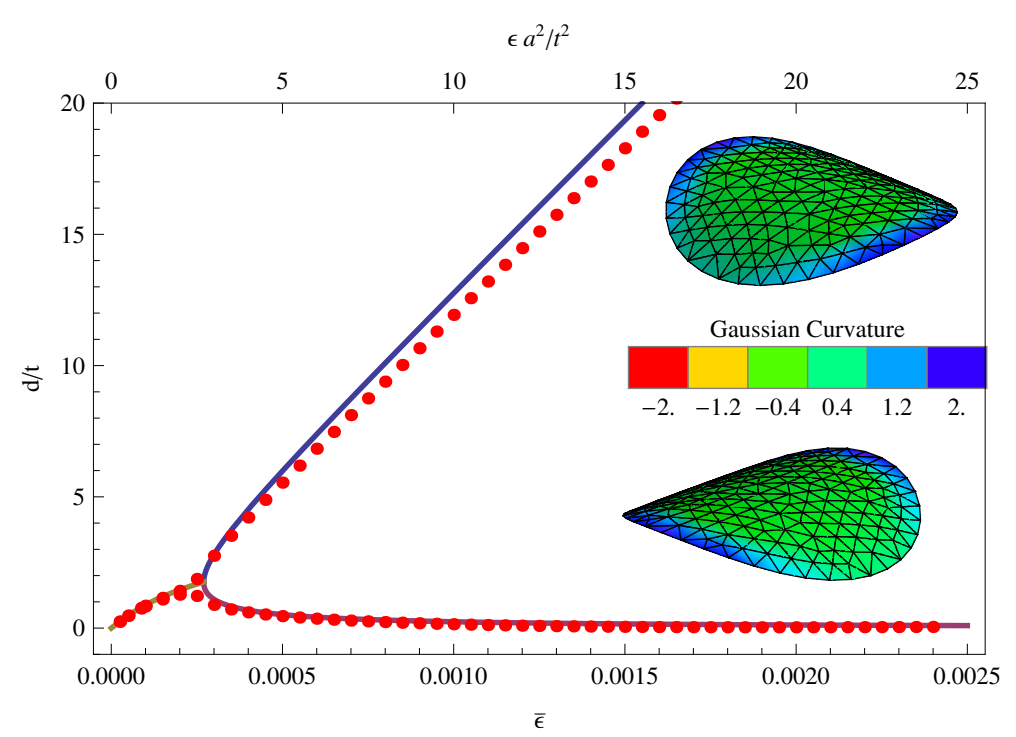

Figure 2: Maximum and minimum edge displacements for equal-sense bending growth of a circular disk with the same properties as in Fig. 1. The load-control parameter, $\bar{\epsilon}$, is defined within the growth strain profile of Eqn 21. The solid lines are theoretical predictions derived from Eqns 19 and 20 before and after buckling, respectively, with the upper curve referring to displacements under $\bar{\kappa}_{x}$, and the lower to $\bar{\kappa}_{y}$ : the bifurcation around $\bar{\epsilon}=0.3 \times 10^{-3}$ signifies buckling, which is also present in the finite element results, given by the dots. The bistable shapes are taken from a point in the post-buckling simulation, and show a considerably reduced degree of Gaussian curvature compared to Fig. 1. Again, there are boundary condition edge effects. 


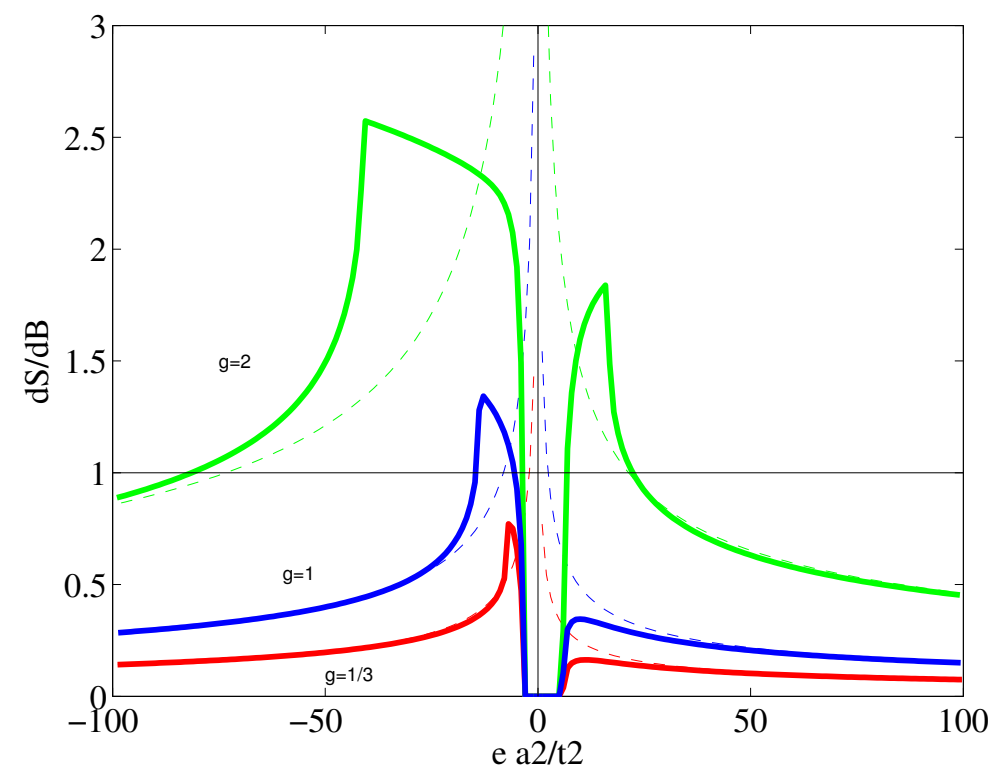

Figure 3: Ratio of maximum displacements of a circular disk during growth, where $d_{\mathrm{S}}$ is the displacement due to extensional growth alone, and $d_{\mathrm{B}}$ is due to bending alone. The specific growth strain distributions are given by Eqn 24 in which $\bar{\epsilon}$ is a common maximum growth strain. The disk has the same properties as in the previous figures, and the solid lines are for different values of $\gamma$, which measures the effectiveness of bending growth: the green highest lines occur when $\gamma$ takes its largest theoretical value of 2, which is assumed in the finite element analysis of Fig. 2; the blue intermediate lines have $\gamma=1$; and the red lower lines have $\gamma=1 / 3$. The dashed lines are derived from simpler asymptotic conditions, and close to the origin displacements are zero before buckling. Portions of any curve which lie above the horizontal line of unity ratio, signify higher displacements during extensional growth, and vice versa. 


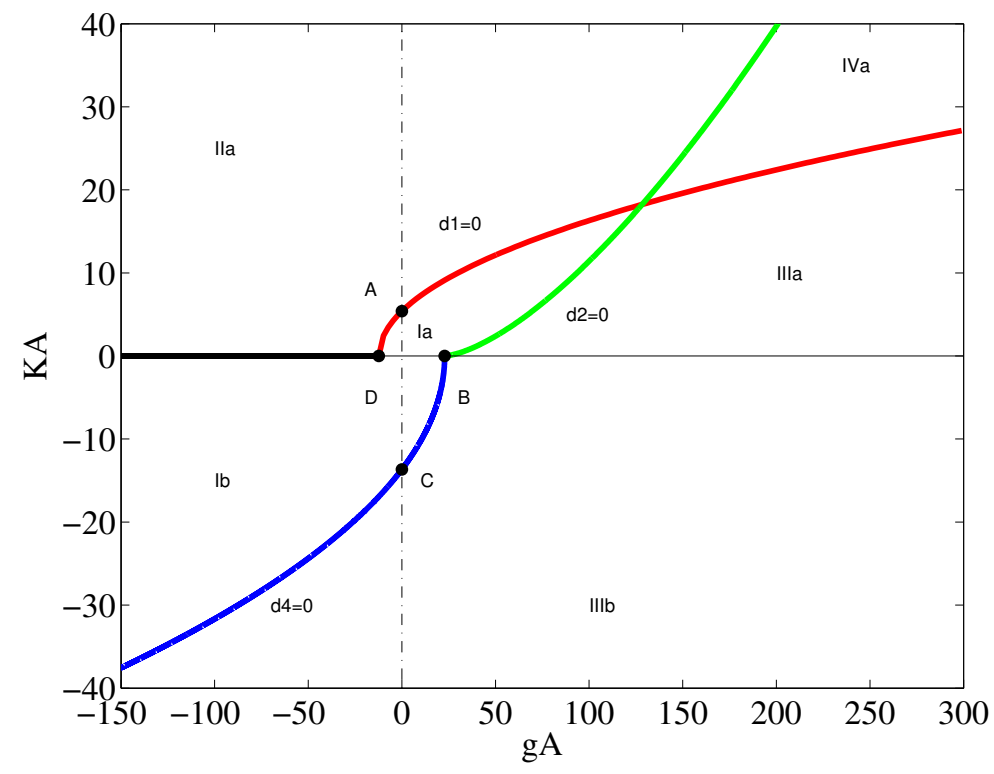

FiguRE 4: Stability performance according to independent extensional (abscissa) and bending (ordinate) growth modes for a circular disk of the same properties as in the previous figures. The ordinate shows the magnitude of $\bar{\kappa}_{x \mathrm{~A}}$ when there is equal-sense bending growth $\left(\bar{\kappa}_{x \mathrm{~A}}=\bar{\kappa}_{y \mathrm{~A}}\right)$, which is plotted upwards, and when there is opposite-sense bending growth $\left(\bar{\kappa}_{x \mathrm{~A}}=-\bar{\kappa}_{y \mathrm{~A}}\right)$, which is plotted downwards. In the top half, the discriminants, $d_{1}$ (red) and $d_{2}$ (green) from Eqn 26, apply and are set equal to zero to reveal the solid loci, and in the bottom half, the same is performed for $d_{4}$ (blue) from Eqn 27: a final boundary (black) separates opposite- and equal-regions behind the intercept of $d_{1}=0$. Four regions are then identified around these loci, and labelled I to IV, with further labels, a and $\mathrm{b}$, for equal- and opposite-sense bending growth, respectively. Region I conforms to monostable disk shapes; region III is always bistable; region II is either bistable if the shear modulus is larger than the isotropic value, otherwise it is neutrally stable for $\alpha=(1-\nu) / 2$; region IV always has shapes that combine the properties of regions II and III. The four single-mode buckling parameters are identified as A to D, and correspond to the formulae for $\bar{\kappa}_{x \mathrm{~A}}^{*}$ and $\bar{g}_{\mathrm{A}}^{*}$ of Section 4 . Note that when $\alpha$ is less than its isotropic value, different solution regimes emerge, which are not treated here.

A

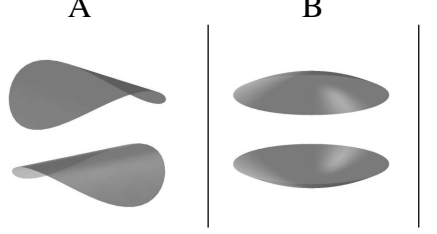

$\mathrm{C}$

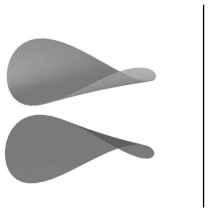

$\mathrm{D}$

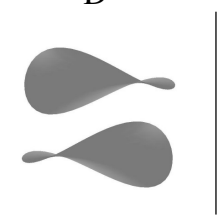

IV.a

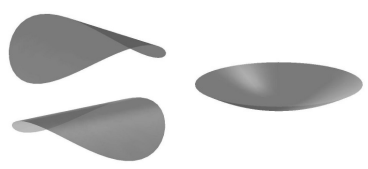

Figure 5: Bistable disk shapes associated with each of the buckling points, A-D, in Fig. 4. The corresponding values of extensional and bending growth strains, $\left(\bar{g}_{\mathrm{A}}^{*}, \kappa_{x \mathrm{~A}}^{*}\right)$, from Section 4 are: A $\left(0, \sqrt{4(1-\nu) / \phi(1+\nu)^{2}}\right) ; \mathrm{B}([1+\nu] / \phi, 0)$; $\mathrm{C}\left(0, \sqrt{4(1+\nu) / \phi(1-\nu)^{2}}\right)$; D $(-[1-\nu] / \phi, 0)$. In region IV.a, there can be tristable behaviour in which the disk is nearly cylindrically curved in orthogonal directions, or cap-like and inverted. When there is material isotropy, bistability is lost in some regions, and the shapes in A and D are neutrally stable, along with the two cylindrical forms from IV.a. 


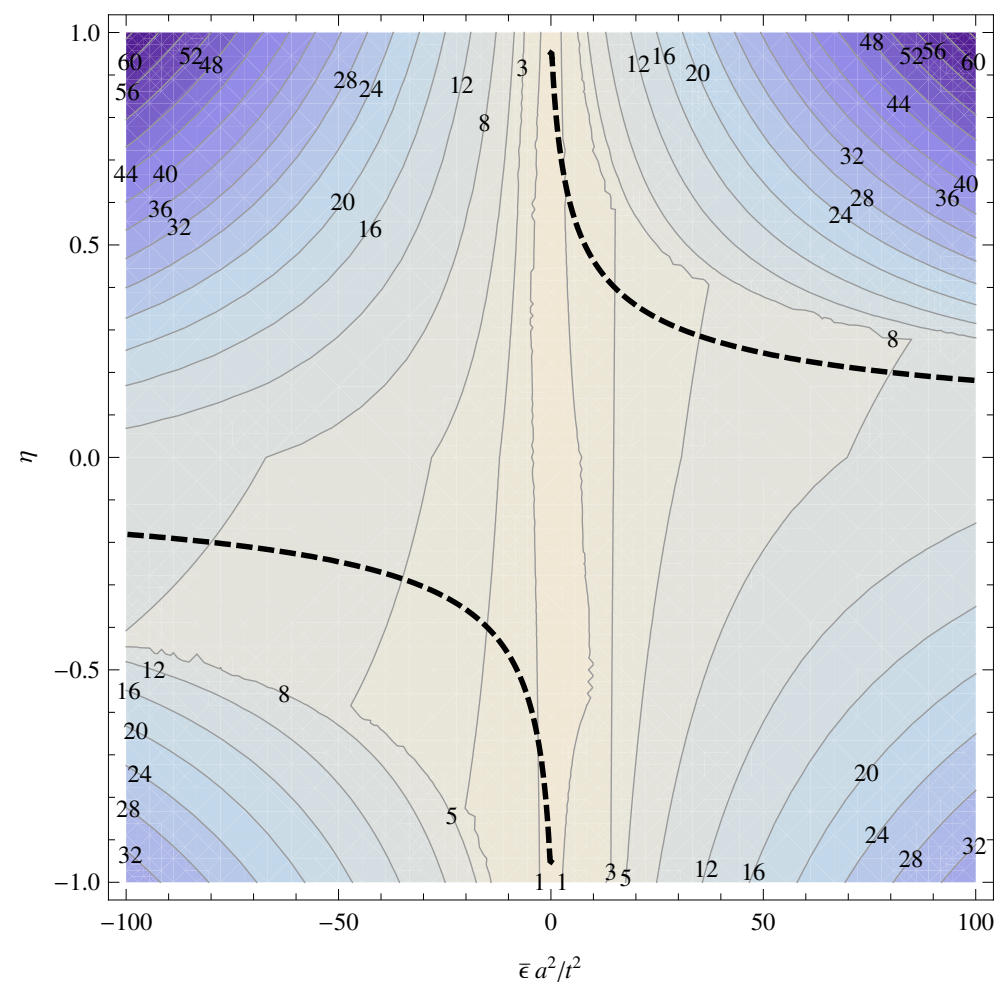

Figure 6: Contours of the ratio of the largest possible displacement to disk thickness during mixed-mode growth. The largest material strain, $\bar{\epsilon}$, has the same physical meaning as in earlier figures: here, a positive value corresponds to positive extensional growth and vice versa. The degree of contribution from either mode is specified as a linear weighted sum through $\eta$ such that $\eta=0$ implies only extensional growth and $\eta= \pm 1$ gives purely bending growth with equal-sense behaviour governed by the range, $0<\eta<1$ and opposite-sense growth belonging to $-1<\eta<0$. Displacements are largest in the corners, when the growth mode is dominated by bending strains, and for small values of $\bar{\epsilon} a^{2} / t^{2}$, the displacement ratio is not very sensitive to $\eta$. The dotted lines are defined in Fig. 7 . 


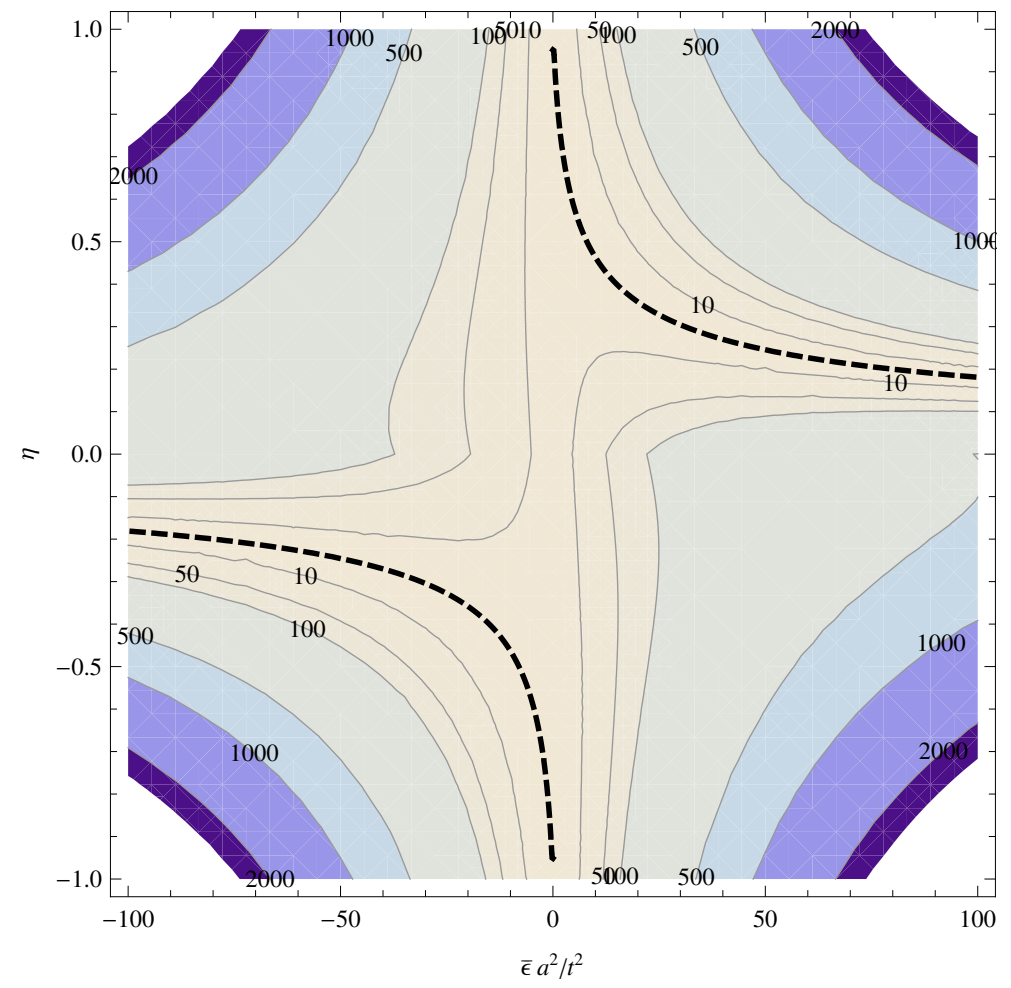

Figure 7: Contours of the lowest amount of strain energy stored in the disk during mixed-mode growth. The coordinate axes are the same as in Fig. 6. The strain energy generally rises with increasing $\bar{\epsilon}$ and in favour of bending growth. The dotted lines are contours of zero stored energy and conform to natural growth shapes. 